\title{
Marsh foraminifera of Prince Edward Island: Their recent distribution and application for former sea level studies
}

\author{
David B. Scott, Mark A. Williamson and Thomas E. Duffett \\ Department of Geology, Dalhousie University, Halifax, N.S. B3H $3 J 5$
}

\begin{abstract}
A detailed survey of all marsh areas of Prince Edward Island, Canada, was undertaken and the information derived was used to determine four optimal areas (i.e., thickest marsh deposits) for sea level studies. Although extensive studies of marsh foraminifera have been conducted in Nova Scotia, the mixed tidal system in Prince Edward Island necessitated further investigations which suggested different relationships in some foraminiferal distributions, possibly linked to the tidal regime of the Gulf of St. Lawrence. Plant species distributions were markedly different, indicating that plant remains, even if preserved, would not be suitable sea level indicators.

Using marsh foraminiferal zonations in subsurface sediments, four sea level curves were determined. These curves encompass the last 3000 years of submergence on Prince Edward Island. Average rates of relative sea level rise in the east (14-19 cm/century) were almost twice that observed in the west $(8 \mathrm{~cm} /$ century). This contrasts with previous work that suggested the island had been subsiding at a uniform rate for the last 3000 years. The data obtained here helps to calibrate recently derived geophysical models of the earth's response following deglaciation.

Taxonomically, a new genus of marsh foraminifera (Pseudothuramina n. gen. Scott, Medioli and Williamson) has been proposed with the type species being Thuramina (?) limnetis, Scott and Medioli described from marsh sediments in Nova Scotia.
\end{abstract}

Un examen en détail de toutes les surfaces marécageuses de I'Ile-du-Prince-Edouard, Canada, a été entrepris, et l'information recueillie a servi à identifier quatre régions optimales (c.-à.-d., dépôts de marais les plus épais) dans le cadre d'études du niveau de la mer. Bien qu'en Nouvelle-Ecosse les foraminifères de marais aient été le sujet d'études poussées, le système de marées mixtes de l'Ile-du-Prince-Edouard a nécessité une étude plus approfondie. Cette étude révèle qu'il existe peut-être des différences de rapport entre certaines répartitions de foraminifères, liées au régime des marées du Golfe du Saint-Laurent. Les répartitions des espèces végétales êtaient très différentes, indiquant que les débris de plantes, même si préservés, ne conviendraient pas comme indicateurs de niveau marin.

Quatre courbes de niveau marin ont été déterminées en employant la distribution par zones des foraminifères dans les sédiments sous-jacents. Ces courbes embrassent les 3,000 dernières années de submersion sur l'Ile-du-Prince-Edouard. Les taux moyens d'élévation du niveau de la mer à l'est (14 à $19 \mathrm{~cm} / \mathrm{siècle)} \mathrm{étaient} \mathrm{presque} \mathrm{le} \mathrm{double} \mathrm{de} \mathrm{ceux} \mathrm{observés} \mathrm{à} \mathrm{l'ouest}(8 \mathrm{~cm} / \mathrm{siècle})$. Ceci contraste avec des travaux antérieurs qui suggéraient un affaissement uniforme de 1 'ile depuis 3,000 ans. Ces données aident à étalonner de récents modèles géophysiques qui simulent la réaction de la terre suite à la déglactiation.

Quant à la taxonomie, on a proposé un nouveau genre de foraminifère de marais (Pseudothurammina n. gen. Scott, Medioli et Williamson) dont l'espèce type est Thuramina (?) Zimmetis, décrite par Scott et Medioli à partir de sédiments de marais de la Nouvelle-Ecosse.

[Traduit par le journal]

\section{INTRODUCTION}

The general effects of Holocene relative sea level rise on Prince Edward Island (hereafter referred to as P.E.I.) are fairly well known. It has been realized for some time that P.E.I. is in a critical position for the study of the response of land masses following deglaciation since one end (the east end) appears to have experienced more relative sea level rise than the other (Kranck 1972). Additionally, raised marine

MARITIME SEDIMENTS AND ATLANTIC GEOLOGY $17,98-129$ (1981) features occur in the west but not the east end of P.E.I. (Prest 1973). The object of our investigation is to provide a detailed framework of information which will aid in the calibration of theoretical models of the earth's response following deglaciation (Peltier and Andrews 1976, Quinlan and Beaumont 1981).

Until recently it was difficult to obtain detailed information on sea level changes because movements of relative sea level in the late Holocene are only in the order of $\mathrm{I}-2 \mathrm{~m}$. Most methods of relocating former sea levels intro- 


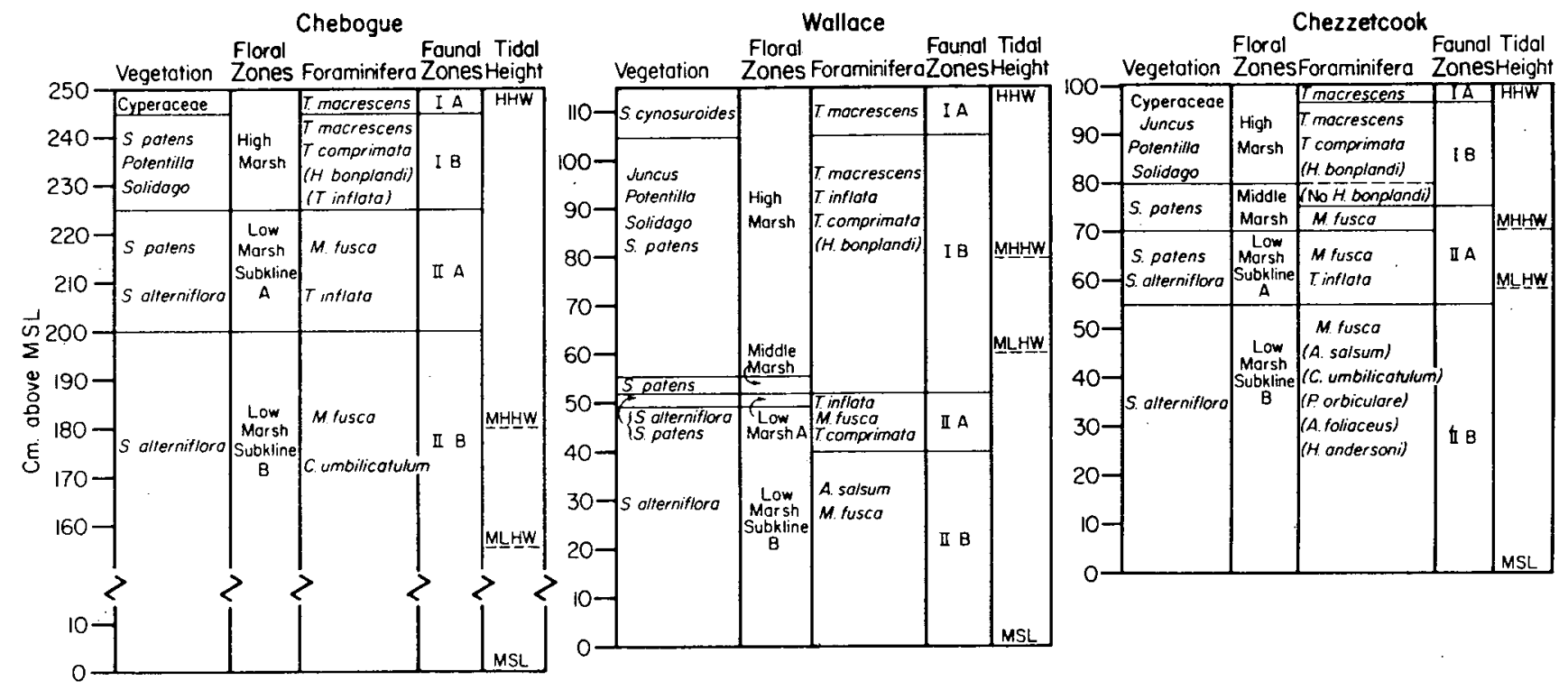

Fig. 1 - Comparative diagram of marsh floral and foraminiferal zones from Nova Scotian marshes (taken from Scott and Medioli, 1980a).

duce errors larger than this (Scott and Medioli 1978a). However, a method has been developed using marsh foraminiferal zonations (Fig. I) that has a potential accuracy of $\pm 5 \mathrm{~cm}$ (Scott 1977; Scott and Medioli, 1978a, 1980a). Factors that limit accuracy are difficulties in measuring during coring or drilling, compaction of sediments, or the absence of zone IA (the zone which marks the area near HHW, Fig. 1). However, if the method is used properly, it is possible to accurately measure the small movements of sea level that have occurred in the last 2000-3000 years.

A large data base is available on marsh foraminifera from nearby Nova Scotia (Scott and Medioli $1980 \mathrm{a})$, however, the tidal regime in the Gulf of st. Lawrence is significantly different (mixed vs semi-diurnal) than that of Nova Scotia which could alter relationships observed in P.E.I. Since no foraminiferal distribution data existed from P.E.I., detailed transects such as those from Nova Scotia were obtained at three 10cations and less detailed information from another. These data can be used for comparison with areas of more normal tidal regimes, increasing the reliability of the sea level work on P.E.I.

Subsequent to obtaining data on surface distribution of foraminifera it was then necessary to locate suitable marsh deposits (i.e. thick enough) for the study of sea level changes. Prest (1973) observed marsh thicknesses up to $5 \mathrm{~m}$ but no precise locations were shown. Hence a detailed exploration of all P.E.I. marshes was undertaken to optimize our detailed drilling effort. Using information obtained in the exploratory phase, four areas were selected for further study:Percival River, Tryon, Pisquid and Orwell (Fig. 2). Using data from these areas it was possible to detect small scale differences in relative sea level rise rates from west to east on P.E.I.

\section{PREVIOUS WORK}

Submergence on P.E.I. was first suggested by Gesner (1846, 1961), Dawson and Harrington (1871) and Johnson $(1913 \mathrm{a}, \mathrm{b})$; however, these early workers had no temporal frame 
of reference since $\mathrm{C}^{14}$ dating had not been developed. Frankel and Crowl (1961) were first to place a date on submerged features on P.E.I., indicating 1.5 to $2.4 \mathrm{~m}$ of submergence in the last 900 years. These data came from Nicholas Point (near orwell, Fig. 2). Kranck (1972), carried out a study of the surficial sediments in the Northumberland strait and inferred a large tilting of the strait (and hence, P.E.I.) relative to present sea level. Deeply submerged features were recognized in the east, and there was progressively less submergence westward. AdditionalIy, emerged marine deposits have been reported in the western end but not in the eastern end of P.E.I. (Owen 1949; Prest 1962, 1973; Dyck and Fyles 1963, 1964). More recently tidal gauge data (Grant $1970 \mathrm{a}, \mathrm{b})$ for Charlottetown indicates submergence during the last 100 years to be $25-30 \mathrm{~cm}$.

Palmer (1974) investigated a mixed marine and freshwater sequence at Basin Head Harbour (near Little Harbour, Site 42, Fig. 2). He suggested sea level rise rates of 3.6 to $10.4 \mathrm{~cm} /$ century during the last 1060 years.

Although no marsh areas have been previousiy examined for foraminifera in P.E.I. , many estuarine areas have been studied. Most work was carried out by G.A. Bartlett or his students at Queen's University, Kingston, Ontario. Studies indicated essentially the same assemblage in all estuaries (dominantly calcareous species) and a complete listing of these reports can be found in scott and others (1980).

METHODS

All samples were collected in June 1978. Detailed surface transects with elevations determined from benchmarks were obtained from Wolfe Inlet (Fig. 3), Mt. Stewart (Fig. 4) and Tryon (Fig. 5). Ad- ditional semi-detailed transects were obtained from Percival River (Fig. 6). Drilling was carried out in Pisquid (Fig. 4), Tryon, Percival River, and Orwell (Fig. 7).

Collection and preparation of surface foraminiferal samples was similar to that by scott and Medioli (1980a). Drill hole sample preparation was similar except that no Rose Bengal or formalin was added, only denatured ethanol. All samples were examined in a water-alcohol mixture.

Exploratory sampling of the subsurface marsh deposits was carried out using a Davis peat corer. This tool can be pushed to the desired depth, triggered, and a small test core retrieved.

Following exploratory testing, detailed drilling was done in those areas with thickest peat sequences. This drilling was carried out with a post-hole auger, a method described by Medioli and scott (1976). Carbon - 14 dates were determined on material obtained only at the base of the drill holes, just above non-compactible substrate to avoid peat compaction problems (Kaye and Barghoorn 1964). Foraminiferal content of the sediment was determined at the dated intervals to establish the exact relative sea level position. This procedure required that several locations be drilled in a transect to obtain an adequate sea level curve. Initially small wood fragments found in the deposits were used for carbon-14 dating; later, however, a whole peat sample was used for dating at orwell because of the scarcity of wood fragments.

\section{RESULTS}

Vegetation and physio-chemical

Vegetation: Vegetation and salinity values for Percival River are summarized in Table 1 while 


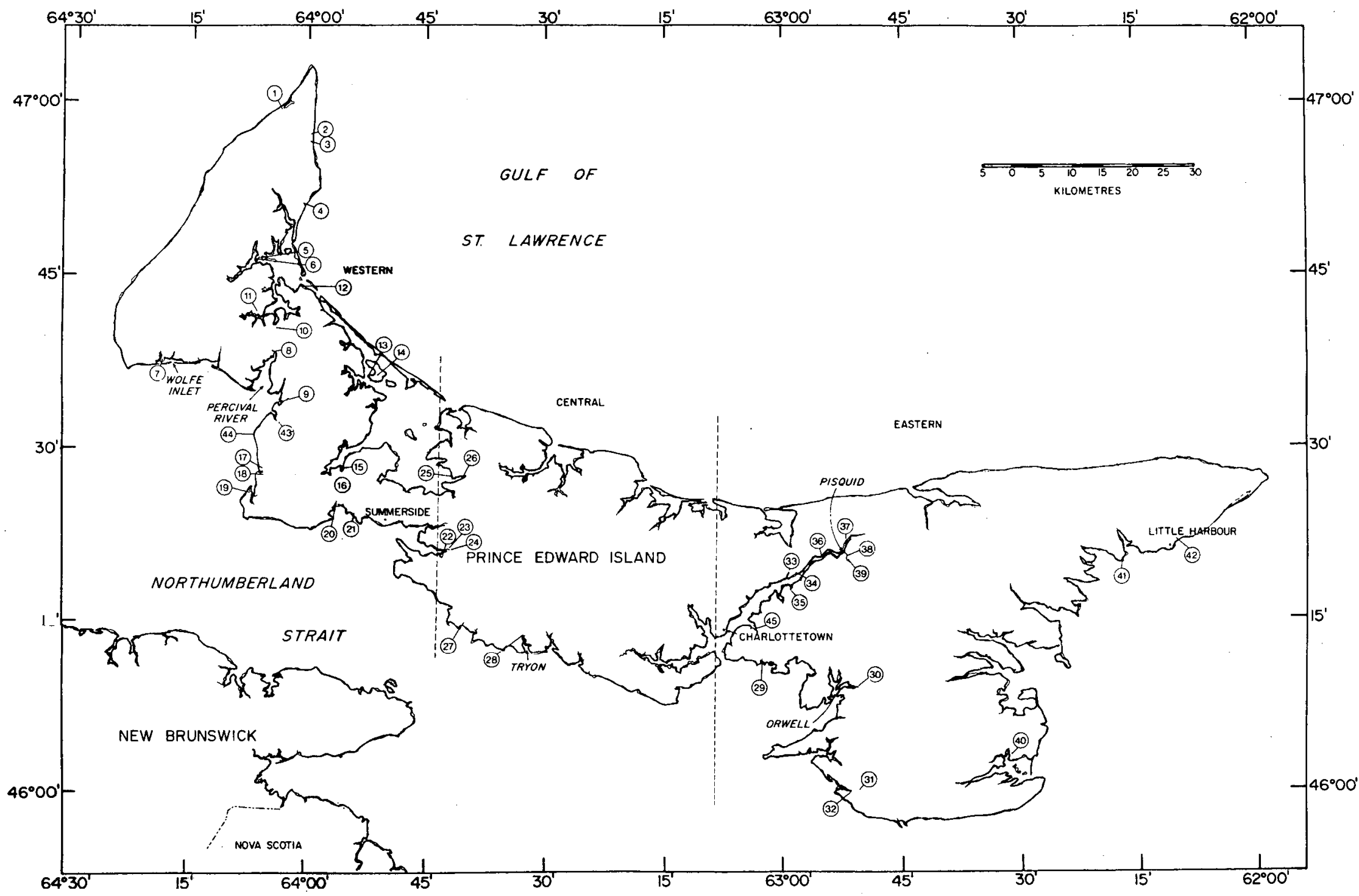

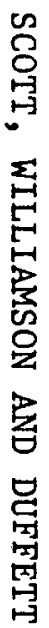


Fig. 2 - Index map indicating all points that were initially investigated (opposite page):

Site ?

Nail Pond marsh $-15 \mathrm{~cm}$ of peat over sand

Site 2

Tignish Harbour marsh - marsh on sand flat $(15 \mathrm{~cm}$ of peat)

Site 3

Little Tignish marsh $-15-180 \mathrm{~cm}$ of peat on sand

Site 4

Foxley peat bog - freshwater peat sample approximately $100 \mathrm{~cm}$ below present mean sea level

Sites $5 \& 6$

Mill River marshes $-90 \mathrm{~cm}$ of peat on sand

Site 7

Wolfe Inlet marsh $-90-120 \mathrm{~cm}$ of peat on sand

Site 8

Percival River marsh $-180-270 \mathrm{~cm}$ of peat on sand

Site 9

Robbs Creek marsh $-60 \mathrm{~cm}$ of peat on sand

Site 10

Portage Bog - $420 \mathrm{~cm}$ of peat - base with freshwater going into sandstone - no marine material

Site 11

Roxbury marsh $-90 \mathrm{~cm}$ of peat on sand

Site 12

Black Banks peat bog - freshwater peat $120 \mathrm{~cm}$ below mean sea leve 1

Site 13

Lennox Isiand, Salt Grass Point $-90 \mathrm{~cm}$ of peat on sand

Site 14

Lennox Island peat bog $-50 \mathrm{~cm}$ of peat on sand

Site 15

Ellis River marsh $-90-120 \mathrm{~cm}$ of peat on sand

Site 16

Miscouche peat bog $-210-270 \mathrm{~cm}$ of freshwater peat going into sand - no marine material

Sites $17 \& 18$

Jacques River marshes $-90-150 \mathrm{~cm}$ of peat on sand

Site 19

Halidimand River marsh $-30 \mathrm{~cm}$ of peat on sand

Sites $20 \& 21$

Sunbury Cove marshes $-0-30 \mathrm{~cm}$ of peat on sand
Site 22

Bedeque marshes $-240-300 \mathrm{~cm}$ of peat on sand

Site 23

Central Bedeque marshes (west of causeway) $-600 \mathrm{~cm}$ of gray mud

Site 24

Central Bedeque marshes (east of causeway) $-450 \mathrm{~cm}$ of peat into sand

Sites $25 \& 26$

Indian River marshes $-30-120 \mathrm{~cm}$ of peat into sand

Site 27

Amherst's Cove marsh $-180 \mathrm{~cm}$ of peat into sand

Site 28

Tryon marsh $-500 \mathrm{~cm}$ of peat on sand

Site 29

Squaw Bay marsh $-90 \mathrm{~cm}$ of peat on sand

Site 30

Orwel1 marsh $-450 \mathrm{~cm}$ in gray mud, $360 \mathrm{~cm}$ in marsh mud into sand

Sites $31 \& 32$

Flat River marshes $-180-300 \mathrm{~cm}$ of mud, some peat into sand

Sites $33 \& 34$

Tenmile House marshes $-90-270 \mathrm{~cm}$ of peat on sand

Site 35

Glenfinnan marsh $-90 \mathrm{~cm}$ of peat on clay or sand

Site 36

Scotchfort marshes $-270 \mathrm{~cm}$ of peat on sand

Site 37

Mt. Stewart (west side of river) marshes $-270-300 \mathrm{~cm}$ of peat on sand

Sites $38 \& 39$

Pisquid marshes $-360-420 \mathrm{~cm}$ of peat on sand

Site 40

Murray Harbour north marsh $-30 \mathrm{~cm}$ of peat on sand

Site 41

Souris - Norris Pond marsh $-15 \mathrm{~cm}$ of peat on sand

Site 42

Little Harbour marsh $-15 \mathrm{~cm}$ of surface peat on sand

Site 43

Victoria West marsh $-90 \mathrm{~cm}$ of peat on sand

Site 44

Rock Point marsh $-30 \mathrm{~cm}$ of peat on sand

Site 45

Fullerton's marsh $-90 \mathrm{~cm}$ of peat on sand 


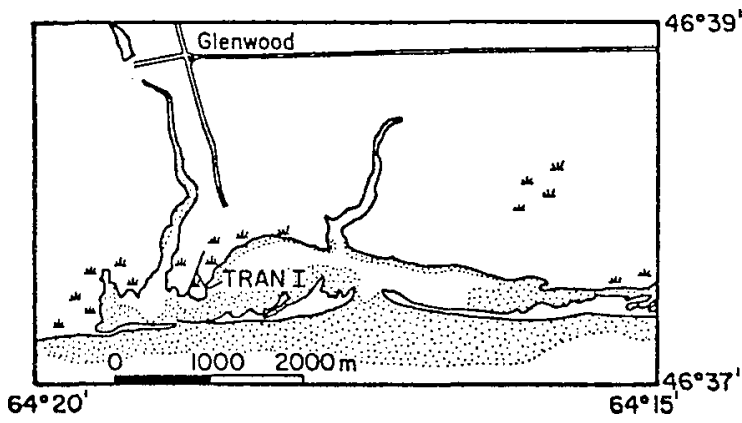

Fig. 3-Map of Wolfe Inlet showing position of the transect.

data from other areas can be found in scott and Medioli (1978b). Plant species are similar to those observed in Nova Scotian marshes (Scott and Medioli 1980a); however, vertical ranges appeared to differ significantly. At Wolfe Inlet, Percival River and Tryon, the middle marsh species Spartina patens, appeared to dominate at all but the lowest levels of the marsh, including supra-tidal areas. At Wolfe Inlet and Percival River Spartina cynosuroides, typically a supra-tidal species, extended into the high marsh zone. Typical

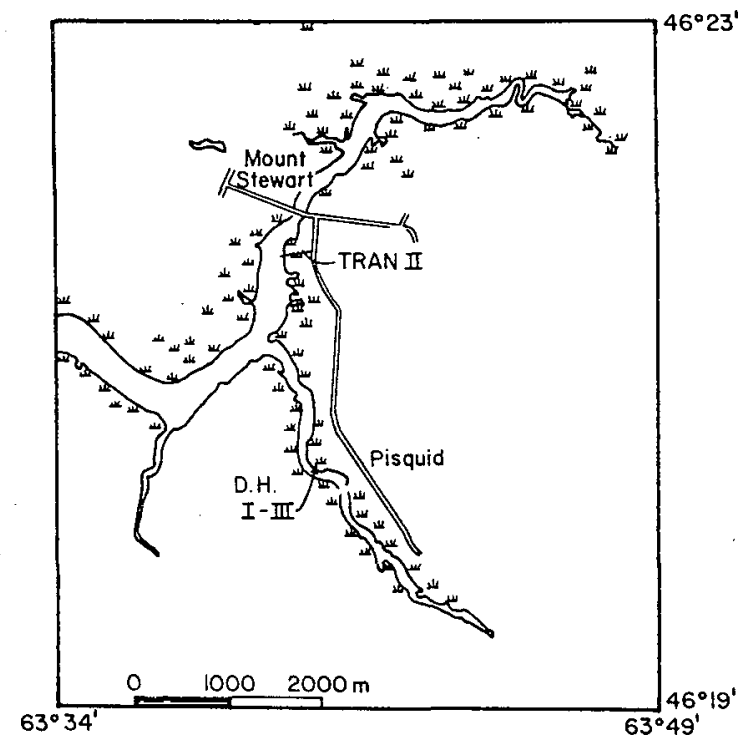

Fig. 4-Map of the Pisquid-Mt. Stewart sampling area. Note causeway landward of the Mt. Stewart transect. high marsh species (i.e. the Cyperaceae and Juncus) were only prominent at one study area, Mt. Stewart.

Salinity: Salinites followed the normal pattern for temperate marsh areas, increasing with decreasing elevation (Scott and Medioli 1978b). At Wolfe Inlet and Percival River salinites were abnormally low, probably because of precipitation that occurred just prior to collection.

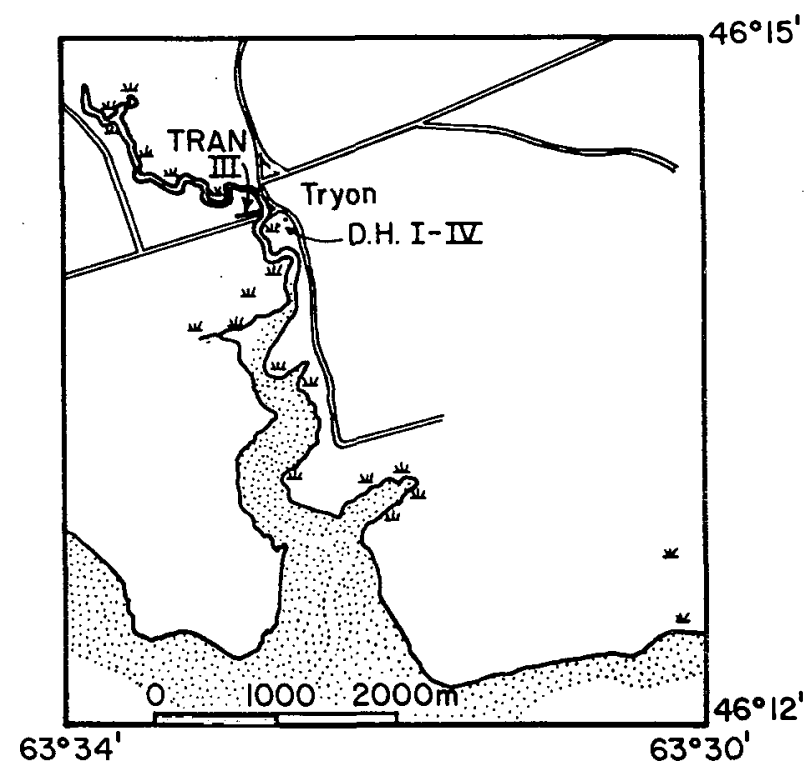

Fig. 5 - Map of Tryon sampling sites; note causeway just seaward of transect. Also, note the strong meander patterns of the Tryon marsh channels.

Tidal factors: Tidal gauge data were available for sites at or near all transect locations and displayed a significant range from east to west. At West Foint (close to Wolfe Inlet and Percival River) total tidal range is given as 161 $\mathrm{cm}$ with higher high water (HHW) at +128 , lower low water (LLW) at $-33 \mathrm{~cm}$ and $\mathrm{Z}_{0}$ at $+67 \mathrm{~cm}$ (note position of $Z_{0}$ with respect to total tidal range). At Victoria (close to Tryon) total tidal range is $290 \mathrm{~cm}$ with $\mathrm{HHW}$ at $+274 \mathrm{~cm}$, LLW at $-16 \mathrm{~cm}$ and $\mathrm{Z}_{0}$ at $+156 \mathrm{~cm}$. At Charlottetown (same as that at Mt. Stewart, Pisquid, Orwell) 
TABLE 1

SALINITY AND VEGETATION TYPES AT PERCIVAL RIVER STATIONS

\begin{tabular}{|c|c|c|c|c|c|c|}
\hline SUB & STATION & STATION & 1 & 2 & 3 & 4 \\
\hline & A & $\begin{array}{l}\text { Plants } \\
\% \text { o }\end{array}$ & $T, J, M, S C$ & $\begin{array}{c}S C \\
0\end{array}$ & $J, S, P A$ & $S C,{ }_{0}^{S P}$ \\
\hline & B & $\begin{array}{l}\text { Plants } \\
\% \%\end{array}$ & $J, \underset{0}{S C}$ & $J, \underset{0}{S C}$ & $S P, S, \underset{0}{P A}$, & $S, S P, \underset{0}{J}, P A$ \\
\hline & c & $\begin{array}{l}\text { Plants } \\
\% \%\end{array}$ & $J, S P_{0} P A$ & $S P, J, S, S C$ & $S P, S$ & $S P, P A$ \\
\hline & $D$ & $\begin{array}{l}\text { Plants } \\
\% \circ\end{array}$ & $S P, P A, J, S, M$ & $S P, S_{0} P A$ & $\stackrel{S P}{7}$ & $S P, P_{4} P A$ \\
\hline & $E$ & $\begin{array}{l}\text { Plants } \\
\% \%\end{array}$ & $S P, \underset{2}{P A}, S A$ & $\begin{array}{c}S P \\
3\end{array}$ & $S A, S P$ & $S, S S, P A, J$ \\
\hline & $F$ & $\begin{array}{l}\text { Plants } \\
\% \%\end{array}$ & $\begin{array}{c}S P \\
8\end{array}$ & $\begin{array}{l}S P \\
9\end{array}$ & $\begin{array}{c}S A \\
6\end{array}$ & $\begin{array}{l}S \\
1\end{array}$ \\
\hline & G & $\begin{array}{l}\text { Plants } \\
\% \% 0\end{array}$ & $S P, \underset{10}{S a}, L$ & $S P, S A$ & & \\
\hline & $H$ & $\begin{array}{l}\text { Plants } \\
\% \% 0\end{array}$ & $S P, \begin{array}{l}S A, L \\
10\end{array}$ & $\begin{array}{l}S A \\
10\end{array}$ & & \\
\hline & I & $\begin{array}{l}\text { Plants } \\
\% \%\end{array}$ & $\begin{array}{l}S A \\
14\end{array}$ & & & \\
\hline & J & $\begin{array}{l}\text { Plants } \\
\% \%\end{array}$ & $\begin{array}{l}S A \\
14\end{array}$ & & & \\
\hline $\begin{array}{l}J- \\
M-1 \\
S C-\end{array}$ & $\begin{array}{l}\text { terrestia } \\
\text { Juncus ge } \\
\text { moss } \\
\text { Spartina }\end{array}$ & $\begin{array}{l}i \\
\text { osuroides }\end{array}$ & $\begin{array}{l}S P \text { - Spart } \\
P A-P o t e r \\
S \text { - Scir } \\
S A-S p a r t\end{array}$ & $\begin{array}{l}\text { patens } \\
\text { a anserina } \\
\text { alterniflora }\end{array}$ & $\begin{array}{l}S a-S o \\
L-L i \\
P-P 2 \\
S S-S o\end{array}$ & ia \\
\hline
\end{tabular}

total tidal range is given as 280 $\mathrm{cm}$ with $\mathrm{HHW}$ at $+280 \mathrm{~cm}$, ILW at 0 $\mathrm{cm}$, and $\mathrm{Z}_{0}$ at $+172 \mathrm{~cm}$.

Tidal regimes in the Gulf of st. Lawrence are mixed (i.e. both diurnal and semi-diurnal components have significant influences). Consequently tidal constants (particularly mean sea level) as determined from tide gauges, are slightly different than for systems with a dominantly semi-diurnal components. Most tidal gauge stations from P.E.I. indicate $\mathrm{Z}_{0}$ (mean sea level or MSL) as occurring in the upper $2 / 5$ of the tidal range rather than in the middle. It appears from our transect studies, however, that benchmark datum (given as MSL) is the midpoint of the tides rather than $Z_{0}$ from the tidal gauges (i.e. the midpoint is not the average level).
Foraminiferal Results Surface Distributions

The surface sample data (Tables 2-9) include percentages of living and total foraminifera. Although numbers of living foraminifera were generally high, they were irregular; hence total populations were used to determine assemblages. Also, it has been demonstrated that total populations best represent prevailing marine conditions, particularly in a marsh (Scott and Medioli 1980b).

In general, 22 species of foraminifera and thecamoebians were recorded from the surface samples, 17 of which had living representatives at the time of collection. Marsh foraminiferal zones and subzones discussed here are those de- 


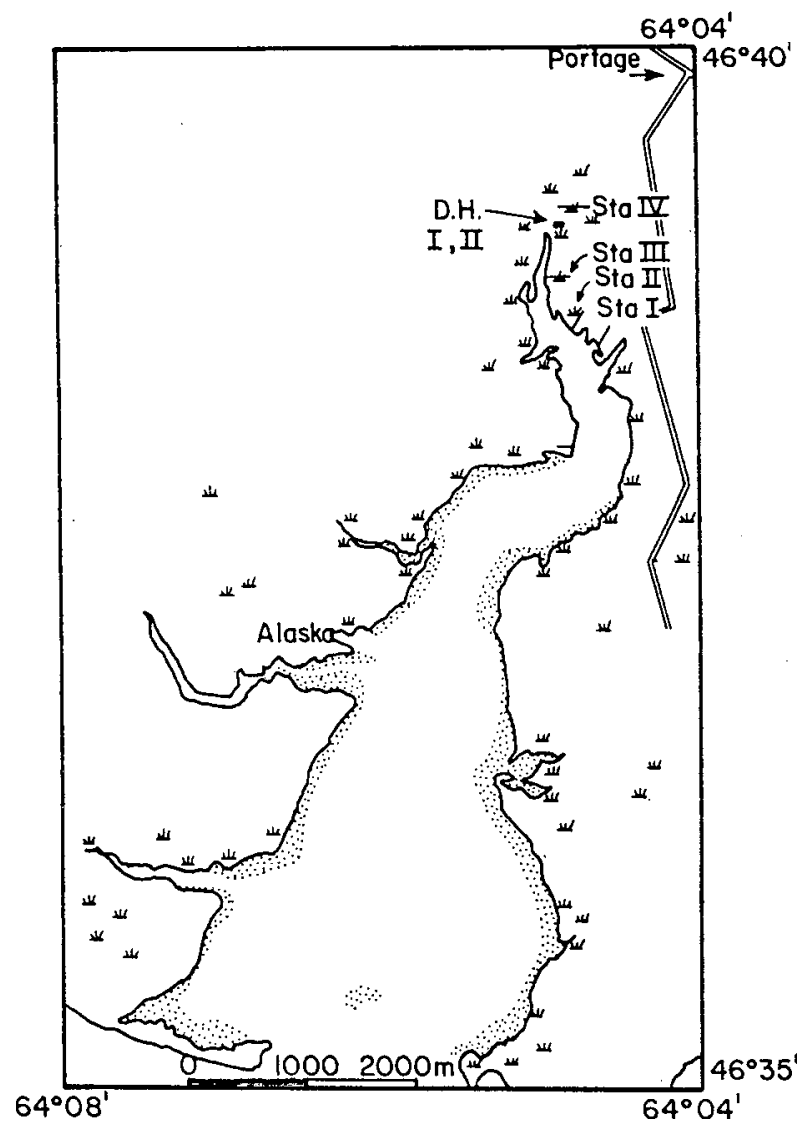

Fig. 6 - Map of Percival River sampling localities.

fined in Scott and Medioli (1978a, 1980a) and have previously been briefly illustrated (Fig. 1).

Wolfe Inlet - Transect I: Foraminifera" dis ribu ions here closely parallel those observed in Nova Scotia (Fig. 8, Tables 2,3). Supratidal areas are characterized by relatively low numbers of the thecamoebian species Centropyxis aculeata togetherwith a few specimens of Trochammina macrescens (Stations 1, 2 Table 2). In the elevation range +88 to $+93 \mathrm{~cm}$ (Fig. 8), foraminiferal zone IA is recognized except that instead of being monospecific with $T$. macrescens (Fig. 1), C. aculeata (a thecamoebian) is also present. Zone IB occurs at +42 to $+75 \mathrm{~cm}$, characterized by co-dominant species T. macrescens and Tiphotro$c^{*}$ a compr ma a; Trochammina inflata increases near the base of this zone. Also, near the base of this zone, significant populations $\left(100-200\right.$ ind. $\left./ 10 \mathrm{~cm}^{3}\right)$ of Polysaccamina ipohalina occur, the first such occurrence reported outside the type locality in southern California (Scott 1976a). In the narrow elevation range +29 to $+39 \mathrm{~cm}$ an assemblage similar to zone IIA occurs, except that Miliammina fusca is not one of the dominant constituents. Below this elevation $M$. fusca dominates together with $T$. inflata and percentage frequencies are reduced for $T$. macrescens. Ammotium salsum has low but sustained percentage occurrences, demonstrating the affinity with zone IIB faunas in Nova Scotia (Fig. 1).

Mt. Stewart - Transect II: A complex distribution pattern was observed in this transect (Fig. 9, Tables 4,5$)$. The upper part of the transect (Stations 1-6, Table 4. is supra-tidal and characterized by low numbers of several thecamoebian species. At $+144 \mathrm{~cm}$ (Station 7) a zone IA fauna occurs; however, total numbers of foraminifera are low. Directly below this, the IB zone is found;

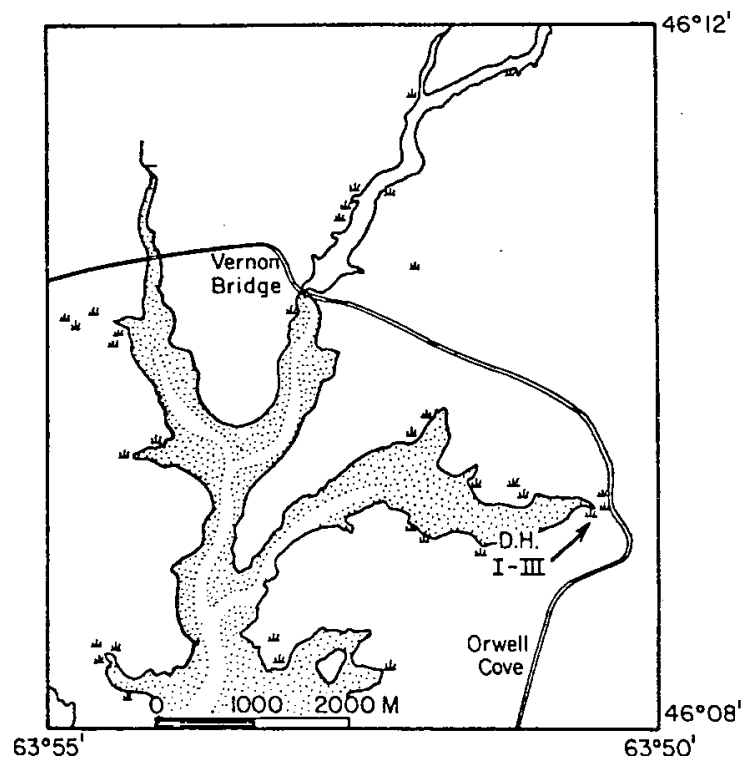

Fig. 7 - Map of Orwell drill holes. 
TABLE 2

FORAMINIFERAL PERCENTAGE OCCURRENCES ALONG WOLFE INLET TRANSECT (STATIONS 1-12)

$\begin{array}{llllllllllllllllllllllllll}\text { STATION NUMBER } & 1 A & 1 B & 2 A & 2 B & 3 A & 3 B & 4 A & 4 B & 5 A & 5 B & 6 A & 6 B & 7 A & 78 & 8 A & 8 B & 9 A & 9 B & 10 A & 10 B & 11 A & 11 B & 12 A & 12 B\end{array}$

$\begin{array}{llllllllllllllllllllllllll}\text { Elevation above MSL ( }(\mathrm{cm}) & 97 & 97 & 98 & 98 & 90 & 90 & 93 & 93 & 88 & 88 & 75 & 75 & 75 & 75 & 74 & 74 & 74 & 74 & 62 & 62 & 56 & 56 & 52 & 52\end{array}$

$\begin{array}{lllllllllllllllllllllllllll}\text { Total species } & \text { Living } & 2 & 1 & 2 & 3 & 2 & 1 & 2 & 2 & 2 & 2 & 2 & 2 & 2 & 2 & 3 & 2 & 2 & 3 & 6 & 2 & 6 & 6 & 4 & 4 \\ & \text { Total } & 3 & 2 & 4 & 4 & 4 & 2 & 2 & 5 & 4 & 3 & 5 & 4 & 6 & 4 & 3 & 6 & 3 & 4 & 6 & 5 & 9 & 8 & 6 & 6\end{array}$

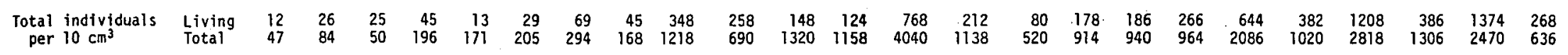

Ammonia beccarii $\quad$ t

Amotium salsum

Arenoparilia mexicana t

Centropyxis aculeata*

Difflugia globulosa

Haplophragmoides

bonplandi

tiliammina fusca

Potysaccamina ipohatina

Reophax nana

Textularia earlandi

Poeudothurantrina

imetis

Tiphotrocha comprimata

Trochammina inflata L

T. macrescens forma
macrescens

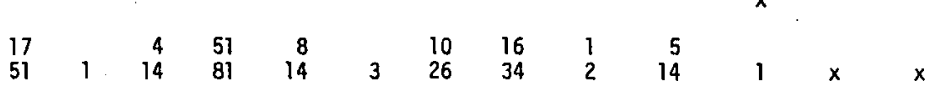

T. macrescens forma
polystoma

$L=$ Live, $T=$ Total, $x=<1 \%$ 
TABLE 3.

FORAMINIFERAL PERCENTAGE OCCURRENCES STATIONS 13-25, WOLFE INLET

$\begin{array}{lllllllllllllllllllllllllll}\text { STATION NUMBER } & 13 A & 13 B & 14 A & 14 B & 15 A & 15 B & 16 A & 16 B & 17 A & 17 B & 18 A & 18 B & 19 A & 198 & 20 A & 20 B & 21 A & 21 B & 22 A & 22 B & 23 A & 23 B & 24 A & 24 B & 25 A & 25 B\end{array}$ \begin{tabular}{lrrrrrrrrrrrrrrrrrrrrrrrrrrrrrrrrr} 
Elevation above MSL (cm) & 49 & 49 & 46 & 46 & 46 & 46 & 42 & 42 & 38 & 38 & 36 & 36 & 29 & 29 & 14 & 14 & 14 & 14 & 7 & 7 & 3 & 3 & -7 & -7 & -23 & -23 \\
Total specíes & $L$ & 3 & 4 & 3 & 2 & 5 & 6 & 5 & 6 & 5 & 5 & 5 & 4 & 7 & 7 & 8 & 8 & 7 & 7 & 2 & 3 & 2 & 6 & 4 & 6 & 4 & 5 \\
\hline & 6 & 7 & 7 & 7 & 8 & 7 & 7 & 8 & 6 & 8 & 7 & 7 & 7 & 9 & 9 & 9 & 8 & 10 & 8 & 8 & 8 & 8 & 9 & 7 & 6 & 8
\end{tabular}

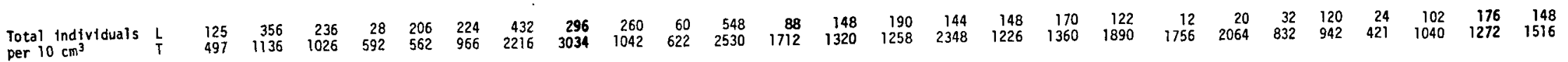
Ammonia beccarii $L$

Annotiven salerm $L$

Arenoparalla

Centropyxis
aculeata

Difflugia

Haplophragmoides

bomplandi

Polysacearmina
ipohalina

Reophax nana

Textularia

earlandi

Pseudothen

$\begin{array}{lll}\text { Timnetis } & \text { T } & \times \\ \text { riphotrocha } & \text { L } & 51 \\ & & 4\end{array}$

Trochamina

Torma macrescens

r. macrescens

forma polystoma

$L=$ Live, $T=$ Total, $x=<$ LIS 
TABLE 4

FORAMiNifERAL PERCENTAGE OCCURRENCES ALONG MT. STEWART TRANSECT (STATIONS 1-13)

\begin{tabular}{|c|c|c|c|c|c|c|c|c|c|c|c|c|c|c|c|c|c|c|c|c|c|c|c|c|c|c|c|c|}
\hline $\begin{array}{l}\text { STATION NUMBE } \\
\text { Elevattion }\end{array}$ & & IA & 18 & $2 \mathrm{~A}$ & 2B & $3 A$ & ${ }_{130}^{38}$ & ${ }^{4 A}$ & $A B$ & $5 \mathrm{~A}$ & 58 & 6A & 68 & $7 \mathrm{~A}$ & 78 & $8 \mathrm{~A}$ & 88 & $9 \mathrm{~A}$ & 98 & $10 \mathrm{~A}$ & 108 & $11 \mathrm{~A}$ & 1178 & ${ }_{12 A}^{12 A}$ & $\begin{array}{l}128 \\
119\end{array}$ & $\begin{array}{l}13 \mathrm{~A} \\
112\end{array}$ & $\begin{array}{l}13 \mathrm{~B} \\
112\end{array}$ & \\
\hline & & & 213 & 184 & 184 & 170 & & 154 & & 158 & 158 & 156 & & 144 & 144 & 135 & 135 & 130 & 130 & 130. & 130 & 130 & 130 & ${ }^{119} 6$ & $\begin{array}{r}179 \\
6\end{array}$ & & & \\
\hline Total species & $\stackrel{t}{T}$ & $\stackrel{0}{i}$ & $\begin{array}{l}0 \\
0\end{array}$ & $i$ & $\frac{1}{3}$ & $\begin{array}{l}1 \\
5\end{array}$ & i & $\frac{1}{4}$ & 1 & $\frac{2}{3}$ & $\begin{array}{l}3 \\
3\end{array}$ & 3 & $\frac{2}{2}$ & $\frac{1}{2}$ & 3 & $\begin{array}{l}2 \\
6\end{array}$ & $\begin{array}{l}5 \\
6\end{array}$ & ${ }_{5}^{4}$ & $\begin{array}{l}4 \\
5\end{array}$ & $\begin{array}{l}4 \\
6 \\
6\end{array}$ & $\begin{array}{l}4 \\
6\end{array}$ & $\begin{array}{l}4 \\
6\end{array}$ & 5 & 6 & 6 & 6 & 5 & \\
\hline $\begin{array}{l}\text { Total individuals } \\
\text { per } 10 \mathrm{~cm}^{3}\end{array}$ & $\stackrel{L}{T}$ & $\stackrel{0}{i}$ & $\begin{array}{l}0 \\
0\end{array}$ & i & $\begin{array}{l}1 \\
4\end{array}$ & $\begin{array}{r}5 \\
16\end{array}$ & 12 & $\begin{array}{l}5 \\
13\end{array}$ & 8 & 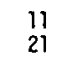 & $\begin{array}{l}17 \\
37\end{array}$ & 26 & $\begin{array}{l}17 \\
28\end{array}$ & 65 & $\begin{array}{l}33 \\
72\end{array}$ & $\begin{aligned} 28 \\
109\end{aligned}$ & $\begin{array}{l}1233 \\
394\end{array}$ & $\begin{array}{l}40 \\
122\end{array}$ & 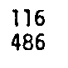 & $\begin{array}{l}73 \\
441\end{array}$ & $\begin{array}{l}238 \\
1078\end{array}$ & $\begin{array}{l}63 \\
262\end{array}$ & $\begin{array}{l}86 \\
346\end{array}$ & $\begin{array}{l}5936 \\
2032\end{array}$ & $\begin{array}{r}8868 \\
3310\end{array}$ & $\begin{array}{r}536 \\
2544\end{array}$ & $\begin{array}{l}130 \\
910\end{array}$ & \\
\hline $\begin{array}{l}\text { Ammobaculites } \\
\text { dizatatus. }\end{array}$ & $\stackrel{t}{T}$ & & & & & & & & & & & & & & & & & & & & & & & & & & & \\
\hline Ammonia beccarii & $\stackrel{L}{T}$ & & & & & & & & & & & & & & & & & & & & & & & & & & & \\
\hline Ammotivon salsim & $\frac{\mathrm{L}}{\mathrm{T}}$ & & & & & & & & & & & & & & & & & & & & & & & & & & & \\
\hline $\begin{array}{l}\text { Arenoparella } \\
\text { mexicanana }\end{array}$ & $\stackrel{L}{T}$ & & & & & & & & & & & & & & & & & & & & & & & & & & & \\
\hline $\begin{array}{l}\text { Centropyxis } \\
\text { aculeata" }\end{array}$ & $\stackrel{\mathrm{t}}{\mathrm{t}}$ & & & & & 16 & & 15 & & & 112 & & & & 3 & 4 & & & & & & & & & & & & \\
\hline $\begin{array}{l}\text { Diffryugia } \\
\text { globurloge }\end{array}$ & $\frac{1}{t}$ & & & & $\begin{array}{c}100 \\
50\end{array}$ & 6 & & 15 & & & $n^{6}$ & 12 & & & & & & & & & & & & & & & & \\
\hline D. oblonga* & $T_{T}^{L}$ & 100 & & & 25 & $\begin{array}{c}100 \\
75\end{array}$ & $\begin{array}{l}100 \\
100\end{array}$ & $\begin{array}{c}100 \\
62\end{array}$ & $\begin{array}{l}100 \\
100\end{array}$ & $\begin{array}{l}91 \\
90\end{array}$ & $\begin{array}{l}82 \\
78\end{array}$ & $\begin{array}{c}100 \\
73\end{array}$ & 64 & 2 & & & & & & & & & & & & & & 3 \\
\hline D. ureoolata* & $\stackrel{t}{T}$ & & & & & & & & & & & & & & & 1 & & $i_{1}^{2}$ & & & & & & & & & & \\
\hline $\begin{array}{l}\text { Baplophragagnoides } \\
\text { bonprabani }\end{array}$ & t & & & & & & & & & & & & & & & 4 & $x^{1}$ & ${ }_{8}^{20}$ & ${ }_{5}^{2}$ & $\begin{array}{l}22 \\
15\end{array}$ & $\begin{array}{l}6 \\
4\end{array}$ & $\begin{array}{l}22 \\
21\end{array}$ & $\begin{array}{l}13 \\
17\end{array}$ & $\begin{array}{l}15 \\
12\end{array}$ & 112 & & ${ }_{9}^{5}$ & \\
\hline Miliarmina fusca & t & & & & & & & & & 5 & & & & & & & $\frac{2}{2}$ & & & 1 & $\mathrm{x}$ & & & $\frac{2}{2}$ & $\frac{2}{5}$ & 19 & 11 & \\
\hline $\begin{array}{l}\text { Polysaccannina } \\
\text { ipohal ina }\end{array}$ & $\stackrel{t}{T}$ & & & & & & & & & & & & & & & 1 & i & & $x$ & $x$ & x & 1 & $x$ & $\stackrel{x}{1}$ & $\underset{x}{x}$ & $x$ & & \\
\hline $\begin{array}{l}\text { Pontigulasaia } \\
\text { compresesa }\end{array}$ & $\stackrel{i}{T}$ & & & & & 6 & & & & & & & & & & & & & & & & & & & & & & \\
\hline $\begin{array}{l}\text { Pereudothuranmina } \\
\text { limnetis }\end{array}$ & $\stackrel{t}{T}$ & & & & & & & & & & & & & & 1 & $\begin{array}{l}71 \\
62\end{array}$ & $\begin{array}{l}55 \\
58\end{array}$ & $\begin{array}{l}42 \\
48\end{array}$ & $\begin{array}{l}499 \\
40\end{array}$ & ${ }_{20}^{33}$ & $\begin{array}{l}44 \\
50\end{array}$ & $\begin{array}{l}10 \\
29\end{array}$ & $\begin{array}{l}13 \\
19 \\
19\end{array}$ & $\begin{array}{l}18 \\
42\end{array}$ & $\begin{array}{l}10 \\
25\end{array}$ & 8 & $10^{2}$ & \\
\hline $\begin{array}{l}\text { Tiphotrocha } \\
\text { camprimata }\end{array}$ & t & & & 100 & 50 & 6 & & 8 & & & & & & & & & 1 & 2 & 1 & $\frac{7}{12}$ & ${ }_{9}^{10}$ & 14 & 望 & $\begin{array}{l}27 \\
15\end{array}$ & $\begin{array}{l}33 \\
19 \\
19\end{array}$ & 17 & ${ }_{21}^{22}$ & \\
\hline 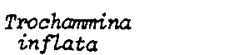 & $\stackrel{t}{T}$ & & & & & & & & & & & & & & & & & & & & & & & & & & & \\
\hline 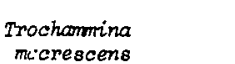 & t & & & & & & & & & $\frac{9}{5}$ & & 15 & 年1 & $\begin{array}{l}100 \\
98\end{array}$ & $\begin{array}{c}100 \\
96\end{array}$ & ${ }_{28}^{28}$ & $\begin{array}{l}44 \\
37\end{array}$ & $\begin{array}{l}35 \\
41 \\
41\end{array}$ & 年 ${ }_{55}^{8}$ & $\begin{array}{l}38 \\
52\end{array}$ & ${ }_{37}^{39}$ & 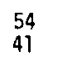 & $\begin{array}{l}46 \\
44\end{array}$ & $\begin{array}{l}38 \\
27\end{array}$ & $\begin{array}{l}42 \\
39\end{array}$ & $\begin{array}{l}82 \\
56\end{array}$ & $\begin{array}{l}71 \\
49\end{array}$ & \\
\hline
\end{tabular}


TABLE 5

FORAMINIFERAL PERCENTAGE OCCURRENCES (STATIONS 14-28) MT. STEWART TRWSECT

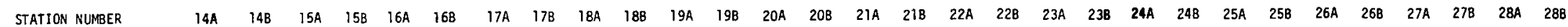
$\begin{array}{llllllllllllllllllllllllllllllll}\text { Elevation above MSL }(\mathrm{cm}) & 111 & 111 & 97 & 97 & 97 & 97 & 91 & 91 & 90 & 90 & 86 & 86 & 91 & 91 & 79 & 79 & 75 & 75 & 37 & 37 & 35 & 35 & 20 & 20 & -25 & -25 & -29 & -29 & -57 & -57\end{array}$ $\begin{array}{llllllllllllllllllllllllllllllllll}\text { Total species } & \mathrm{L} & 5 & 4 & 2 & 4 & 4 & 5 & 6 & 4 & 4 & 3 & 3 & 3 & 4 & 3 & 6 & 4 & 7 & 8 & 7 & 7 & 5 & 6 & 6 & 6 & 6 & 5 & 7 & 5 & 1 & 5 \\ & \mathrm{~T} & 5 & 5 & 3 & 6 & 7 & 7 & 8 & 7 & 6 & 5 & 8 & 4 & 7 & 4 & 8 & 9 & 7 & 8 & 8 & 8 & 7 & 8 & 7 & 8 & 8 & 8 & 9 & 9 & 6 & 9\end{array}$

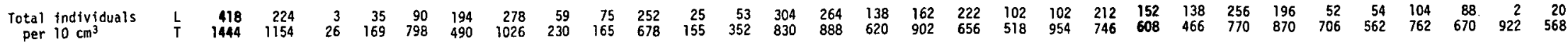
Anmobaculites Anmobacutitation

Amronic beccarii

Armotivan sal sum

Arenoparezla

Centropyzis
aculeata*

Difflugia

D. obtonga*

D. urceolata"

Baplophragnoides

Milianmina fusca

Polyeaccanmina
ipohalina

Pontigulasia
compressa*

Pseudothurarmina

Tiphotrocha
comprimata

Trochormina
inflata

Trochanmina
macrescens
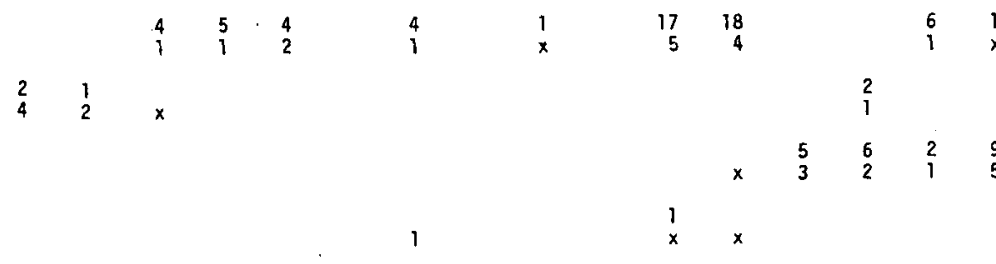

$L=$ Live, $T=$ Total, $x=<18$ 
TABLE 6

FORAMINIFERAL PERCENTAGE OCCURRENCES ALONG. TRYON TRANSECT (STATIONS 1-14)

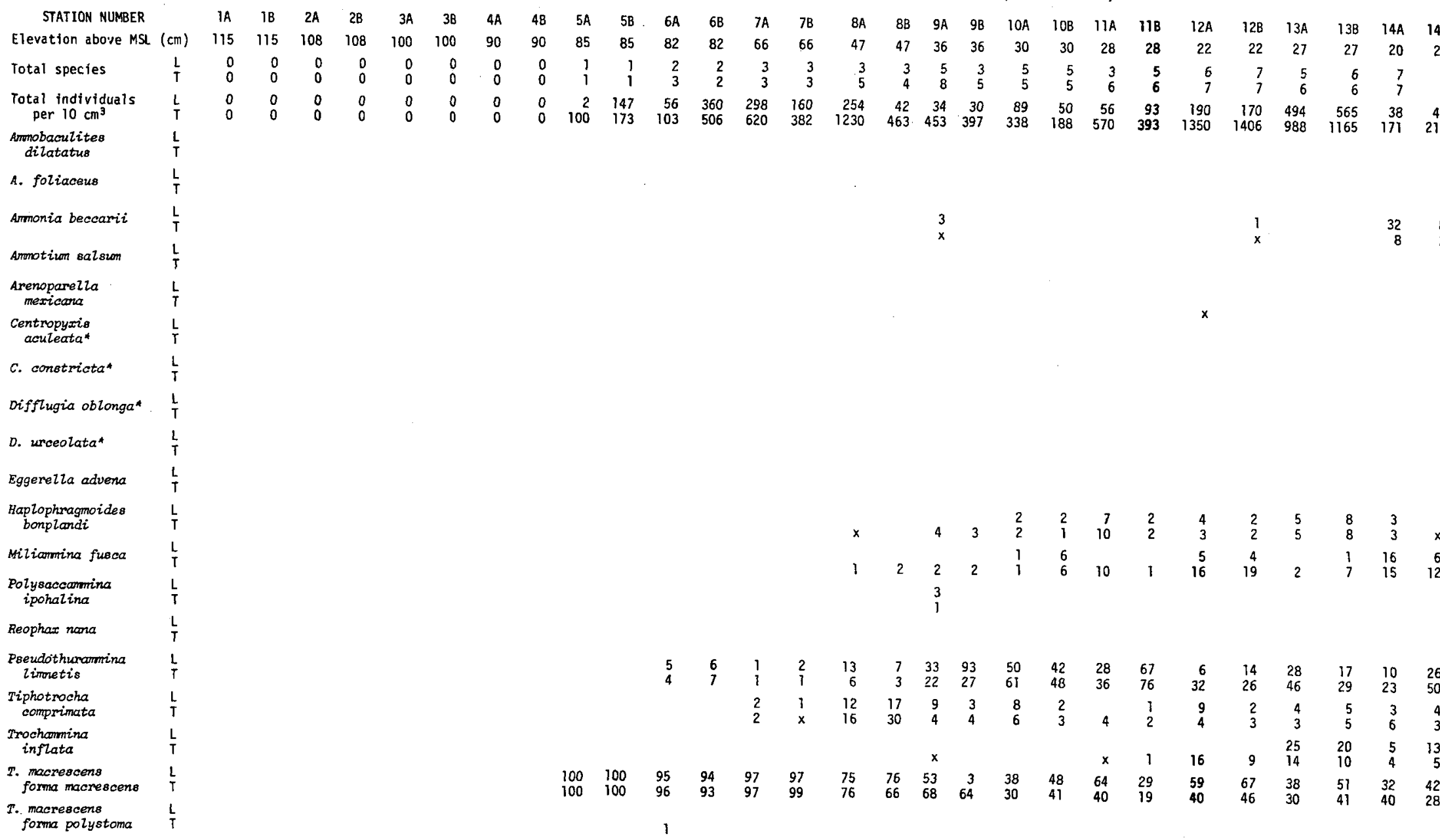


TABLE 7

FORAMINIFERAL PERCENTAGE OCCURRENCES (STATIONS 15-28) TRYON TRANSECT

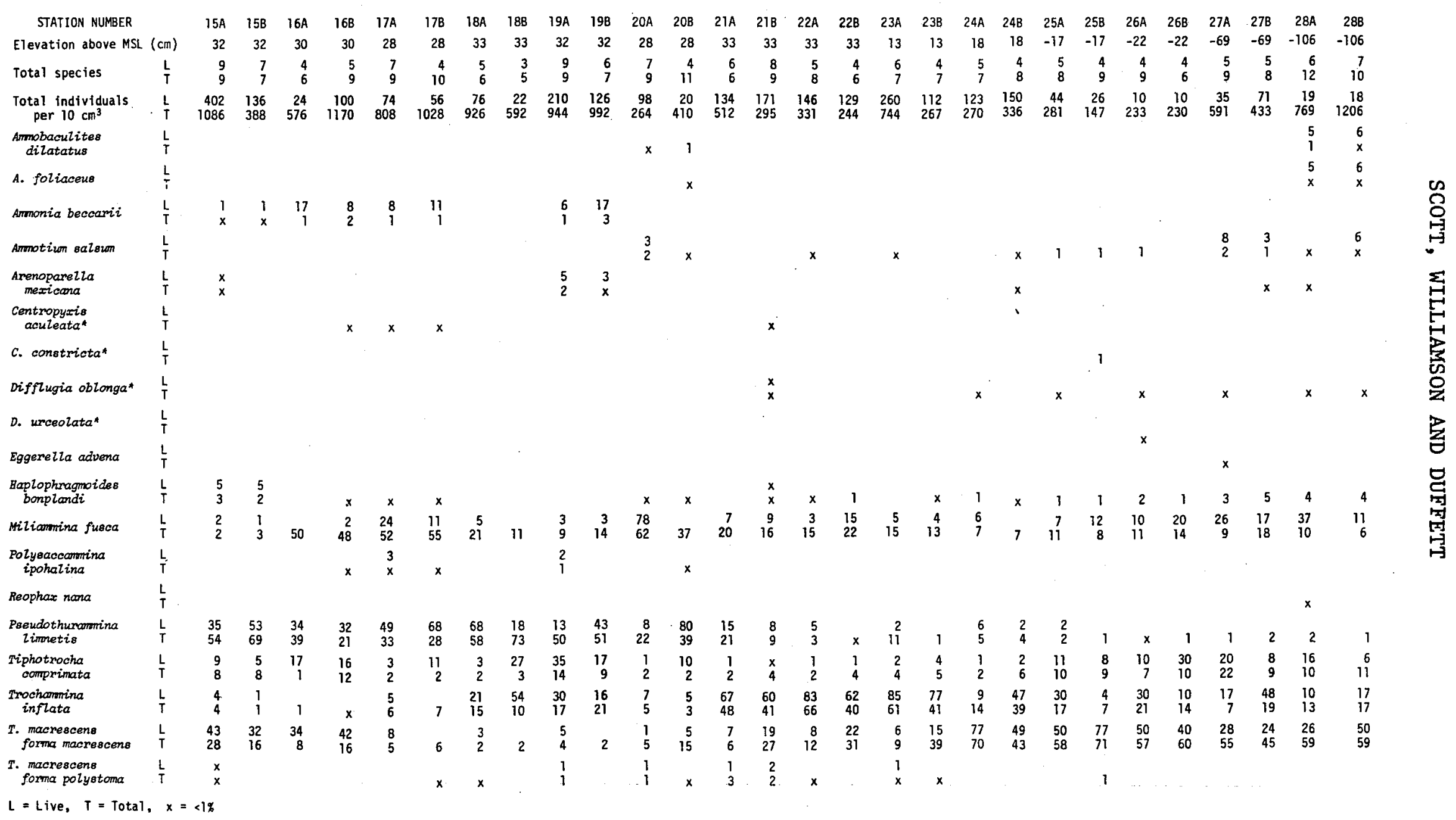


TABLE 8

FORAMINIFERAL PERCENTAGE OCCURRENCES AT PERCIVAL RIVER

(STATIONS la-lj)

\begin{tabular}{|c|c|c|c|c|c|c|c|c|c|c|c|c|c|c|c|c|c|c|c|c|c|}
\hline SAMPLE NO. & & $1 a_{1}$ & $1 a_{2}$ & $1 b_{1}$ & $1 b_{2}$ & $1 c_{1}$ & $i c_{2}$ & $1 d_{1}$ & $i d_{2}$ & $1 e_{1}$ & $1 e_{2}$ & $1 f_{1}$ & $l f_{2}$ & $\lg _{1}$ & $1 g_{2}$ & $\ln _{1}$ & $1 h_{2}$ & $1 i_{1}$ & $1 i_{2}$ & $1 j_{1}$ & $1 j_{2}$ \\
\hline No. of species & $\stackrel{L}{T}$ & $\begin{array}{l}0 \\
2\end{array}$ & $\begin{array}{l}0 \\
0\end{array}$ & $\stackrel{0}{1}$ & $\begin{array}{l}0 \\
0\end{array}$ & $\begin{array}{l}1 \\
2\end{array}$ & $\begin{array}{l}1 \\
1\end{array}$ & $\begin{array}{l}1 \\
2\end{array}$ & $\begin{array}{l}1 \\
2\end{array}$ & $\begin{array}{l}4 \\
4\end{array}$ & $\begin{array}{l}4 \\
4\end{array}$ & $\begin{array}{l}4 \\
4\end{array}$ & $\begin{array}{l}4 \\
4\end{array}$ & $\begin{array}{l}3 \\
4\end{array}$ & $\begin{array}{l}3 \\
5\end{array}$ & $\begin{array}{l}3 \\
5\end{array}$ & $\begin{array}{l}4 \\
5\end{array}$ & $\begin{array}{l}5 \\
6\end{array}$ & $\begin{array}{l}3 \\
5\end{array}$ & $\begin{array}{l}3 \\
5\end{array}$ & $\begin{array}{l}3 \\
5\end{array}$ \\
\hline $\begin{array}{l}\text { No. of individuals } \\
10 \mathrm{~cm}^{3}\end{array}$ & $\begin{array}{l}\mathrm{L} \\
\mathrm{T}\end{array}$ & $\begin{array}{l}0 \\
4\end{array}$ & $\begin{array}{l}0 \\
0\end{array}$ & $\begin{array}{l}0 \\
2\end{array}$ & $\begin{array}{l}0 \\
0\end{array}$ & $\begin{array}{r}41 \\
125\end{array}$ & $\begin{array}{r}58 \\
293\end{array}$ & $\begin{array}{l}179 \\
424\end{array}$ & $\begin{array}{r}36 \\
132\end{array}$ & $\begin{array}{r}49 \\
270\end{array}$ & $\begin{array}{l}122 \\
725\end{array}$ & $\begin{array}{l}108 \\
281\end{array}$ & $\begin{array}{r}98 \\
266\end{array}$ & $\begin{array}{r}38 \\
782\end{array}$ & $\begin{array}{r}310 \\
1056\end{array}$ & $\begin{array}{r}32 \\
569\end{array}$ & $\begin{array}{r}124 \\
1108\end{array}$ & $\begin{array}{r}220 \\
1460\end{array}$ & $\begin{array}{r}34 \\
1104\end{array}$ & $\begin{array}{r}134 \\
1034\end{array}$ & $\begin{array}{r}261 \\
1233\end{array}$ \\
\hline Armotizam salsrom & $\stackrel{L}{T}$ & & & & & & & & & & & & & & & & & & & & l \\
\hline $\begin{array}{l}\text { Arenoparella } \\
\text { mexicana }\end{array}$ & L & & & & & & & & & & & & & & & $\begin{array}{r}19 \\
3\end{array}$ & $\begin{array}{r}16 \\
i\end{array}$ & $\begin{array}{l}5 \\
2\end{array}$ & $\begin{array}{r}65 \\
5\end{array}$ & $\begin{array}{l}7 \\
x\end{array}$ & $\begin{array}{l}7 \\
3\end{array}$ \\
\hline $\begin{array}{l}\text { Haplophragmoides } \\
\text { bonplandi }\end{array}$ & L & & & & & & & & & & & & & & & & & & & & \\
\hline Miliammina fusca & $\begin{array}{l}\mathrm{L} \\
\mathrm{T}\end{array}$ & & & & & & & & & $\begin{array}{l}6 \\
9\end{array}$ & $\begin{array}{l}10 \\
12\end{array}$ & $\begin{array}{l}7 \\
7\end{array}$ & 7 & $\begin{array}{l}16 \\
20\end{array}$ & 27 & $\begin{array}{r}3 \\
45\end{array}$ & $\begin{array}{l}10 \\
55\end{array}$ & $\begin{array}{l}65 \\
84\end{array}$ & $\begin{array}{r}6 \\
48\end{array}$ & $\begin{array}{l}75 \\
85\end{array}$ & $\begin{array}{l}79 \\
77\end{array}$ \\
\hline $\begin{array}{l}\text { Preudothurammina } \\
\text { Iimnetis }\end{array}$ & $\stackrel{t}{T}$ & & & & & & & & & & & & & 4 & $\begin{array}{l}1 \\
2\end{array}$ & & & $\begin{array}{l}x \\
x\end{array}$ & & & \\
\hline $\begin{array}{l}\text { Tiphotrocha } \\
\text { comprimata }\end{array}$ & $\stackrel{L}{T}$ & 75 & & & & 2 & & 2 & 4 & $\begin{array}{l}4 \\
8\end{array}$ & $\begin{array}{l}1 \\
4\end{array}$ & $\begin{array}{l}22 \\
20\end{array}$ & $\begin{array}{l}20 \\
23\end{array}$ & 8 & $\begin{array}{r}6 \\
12\end{array}$ & $\begin{array}{l}3 \\
5\end{array}$ & $\begin{array}{l}1 \\
4\end{array}$ & $\begin{array}{l}x \\
x\end{array}$ & 4 & $x$ & \\
\hline Trochanmina inflata & $\stackrel{L}{T}$ & & & & & & & & & $\begin{array}{r}8 \\
12\end{array}$ & $\begin{array}{l}2 \\
3\end{array}$ & $\begin{array}{l}40 \\
30\end{array}$ & $\begin{array}{l}36 \\
23\end{array}$ & $\begin{array}{l}80 \\
58\end{array}$ & $\begin{array}{l}77 \\
57\end{array}$ & $\begin{array}{l}75 \\
38\end{array}$ & $\begin{array}{l}73 \\
37\end{array}$ & $\begin{array}{l}28 \\
13\end{array}$ & $\begin{array}{l}29 \\
41\end{array}$ & $\begin{array}{l}18 \\
13\end{array}$ & $\begin{array}{l}14 \\
18\end{array}$ \\
\hline T. macrescens & $\begin{array}{l}\mathrm{L} \\
\mathrm{T}\end{array}$ & 25 & & 100 & & $\begin{array}{r}100 \\
98\end{array}$ & $\begin{array}{l}100 \\
100\end{array}$ & $\begin{array}{r}100 \\
98\end{array}$ & $\begin{array}{r}100 \\
96\end{array}$ & $\begin{array}{l}82 \\
71\end{array}$ & $\begin{array}{l}82 \\
81\end{array}$ & $\begin{array}{l}32 \\
43\end{array}$ & $\begin{array}{l}33 \\
47\end{array}$ & $\begin{array}{r}4 \\
10\end{array}$ & $\begin{array}{l}8 \\
8\end{array}$ & 9 & 3 & $x$ & 2 & $\mathrm{x}$ & 1 \\
\hline Thecamoebians & $\begin{array}{l}\mathrm{L} \\
\mathrm{T}\end{array}$ & & & & & & $x$ & & & $x$ & & & & & & & & & & & \\
\hline & & & & & & (ST) & ATIONS & $2 a-2 e$ & & & & & & & & & & & & & \\
\hline SAMPLE NO. & & & & $2 a_{2}$ & $2 a_{2}$ & $2 b_{1}$ & $2 b_{2}$ & $2 c_{1}$ & $2 \epsilon_{2}$ & $2 d_{1}$ & $2 d_{2}$ & $2 \mathbf{e}_{1}$ & $2 e_{2}$ & & & & & & & & \\
\hline No. of species & $\stackrel{\text { L }}{T}$ & & & $\begin{array}{l}1 \\
4\end{array}$ & $\begin{array}{l}0 \\
0\end{array}$ & $\begin{array}{l}1 \\
2\end{array}$ & $\begin{array}{l}0 \\
3\end{array}$ & $\begin{array}{l}1 \\
2\end{array}$ & 1 & $\begin{array}{l}2 \\
5\end{array}$ & $\begin{array}{l}1 \\
5\end{array}$ & $\begin{array}{l}1 \\
6\end{array}$ & $\begin{array}{l}3 \\
7\end{array}$ & & & & & & & & \\
\hline $\begin{array}{l}\text { No. - of individuals } \\
10 \mathrm{~cm}^{3}\end{array}$ & $\stackrel{L}{T}$ & & & $\begin{array}{r}1 \\
13\end{array}$ & $\begin{array}{l}0 \\
0\end{array}$ & $21^{5}$ & $\begin{array}{l}0 \\
6\end{array}$ & $\begin{array}{r}23 \\
131\end{array}$ & $21^{2}$ & $\begin{array}{r}27 \\
161\end{array}$ & $\begin{array}{r}67 \\
392\end{array}$ & $\begin{array}{r}29 \\
463\end{array}$ & $\begin{array}{r}87 \\
351\end{array}$ & & & & & & & & \\
\hline Armotiven salsrm & $\begin{array}{l}\mathrm{L} \\
\mathrm{T}\end{array}$ & & & & & & & & & & & & & & & & & & & & \\
\hline $\begin{array}{l}\text { Arenoparalla } \\
\text { mexicana }\end{array}$ & $\frac{L}{T}$ & & & & & & & & & & & $x$ & $\begin{array}{l}7 \\
2\end{array}$ & & & & & & & & \\
\hline $\begin{array}{l}\text { Haplophragmoides } \\
\text { bonplandi }\end{array}$ & $\begin{array}{l}L \\
T\end{array}$ & & & & & & & & & & & & 1 & & & & & & & & \\
\hline Milidormina fusca & $\stackrel{L}{T}$ & & & 38 & & 14 & 17 & & & 2 & 1 & 4 & 3 & & & & & & & & \\
\hline $\begin{array}{l}\text { Pseudothurammina } \\
\text { linnetis }\end{array}$ & $\begin{array}{l}\mathrm{L} \\
\mathrm{T}\end{array}$ & & & & & & & & & $\begin{array}{l}7 \\
9\end{array}$ & 4 & $x$ & 1 & & & & & & & & \\
\hline $\begin{array}{l}\text { Tiphotrocha } \\
\text { comprimata }\end{array}$ & $\frac{L}{T}$ & & & 8 & & & & & & 4 & 5 & 12 & $\begin{array}{r}3 \\
12\end{array}$ & & & & & & & & \\
\hline Irochammina inflata & $\begin{array}{l}\mathrm{L} \\
\mathrm{T}\end{array}$ & & & $\begin{array}{r}100 \\
38\end{array}$ & & & 17 & 1 & & 5 & 2 & 19 & 9 & & & & & & & & \\
\hline T. macrescens & $\mathrm{L}$ & & & 16 & & $\begin{array}{r}100 \\
86\end{array}$ & 66 & $\begin{array}{r}100 \\
99\end{array}$ & $\begin{array}{l}100 \\
100\end{array}$ & $\begin{array}{l}93 \\
80\end{array}$ & $\begin{array}{r}100 \\
88\end{array}$ & $\begin{array}{r}100 \\
64\end{array}$ & $\begin{array}{l}90 \\
71\end{array}$ & & & & & & & & \\
\hline Thecamoebians & $T$ & & & & & & $x$ & & $x$ & & & & & & & & & & & & \\
\hline
\end{tabular}

however, this zone can be divided into an upper and lower part. The upper part $(+119$ to $+135 \mathrm{~cm})$ is characterized by high numbers of Pseudothurammina limnetis (new genus, formerly Thuramina? limnetis), Trochammina macrescens, and slightly lower percentages of Haplophragmoides bonplandi. Tiphotrocha comprimata occurs in relatively low percentages. The lower part $(+75$ to $+119 \mathrm{~cm})$ is characterized by $T$. macrescens and $T$. comprimata in equal numbers, with reductions in the other species. In the elevation range that generally corresponds with zone IIA $(+20$ to $+75 \mathrm{~cm}) \quad T$. macrescens is reduced in its percentage occurrence, T. comprimata becomes dominant and several species have sustained but low percentage occurrences (Arenoparella mexicana, Miliammina fusca, Trochammina inflatal. The occurrence of A. mexicana here is the first report of this species as a significant part of an assemblage zone in Atlantic 
TABLE 9

FORAMINIFERAL PERCENTAGE OCCURRENCES AT PERCIVAL RIVER

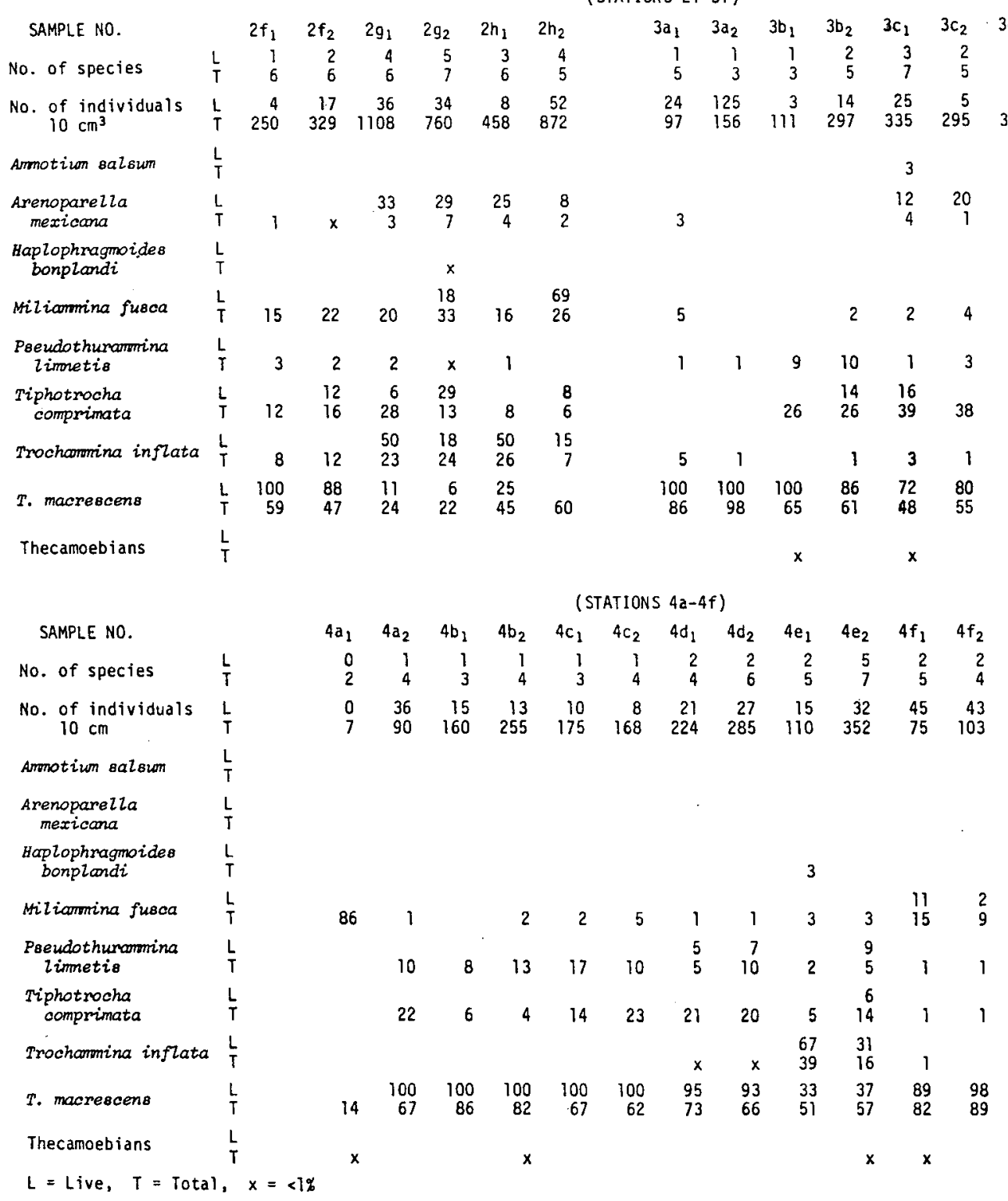

Canada. Below this (-57 to $20 \mathrm{~cm}$ ) Ammotium salsum appears in low percentages while $M$. fusca appears to increase, corresponding with zone IIB.

Tryon - transect iII: Total numbers of foraminifera were generally lower in this transect (Fig. 10 , Tables 6,7 ) and mineral content of the sediments higher, perhaps indicating a higher sedimentation rate. The upper part of the transect contained no forami- nifera or thecamoebians (sediment was generally very ary). At +85 $\mathrm{cm}$ a monospecific fauna of Trochammina macrescens (Zone IA) occurs. Below this (+28 to $+82 \mathrm{~cm})$ a zone IB fauna occurs, co-dominated by Pseudothurammina 2 imnetis and $T$. macrescens, with small numbers of Tiphotrocha comprimata. Below this elevation, Trochammina inflata and Miliammina fusca become more prominent. At the seaward end of this transect (i.e. 


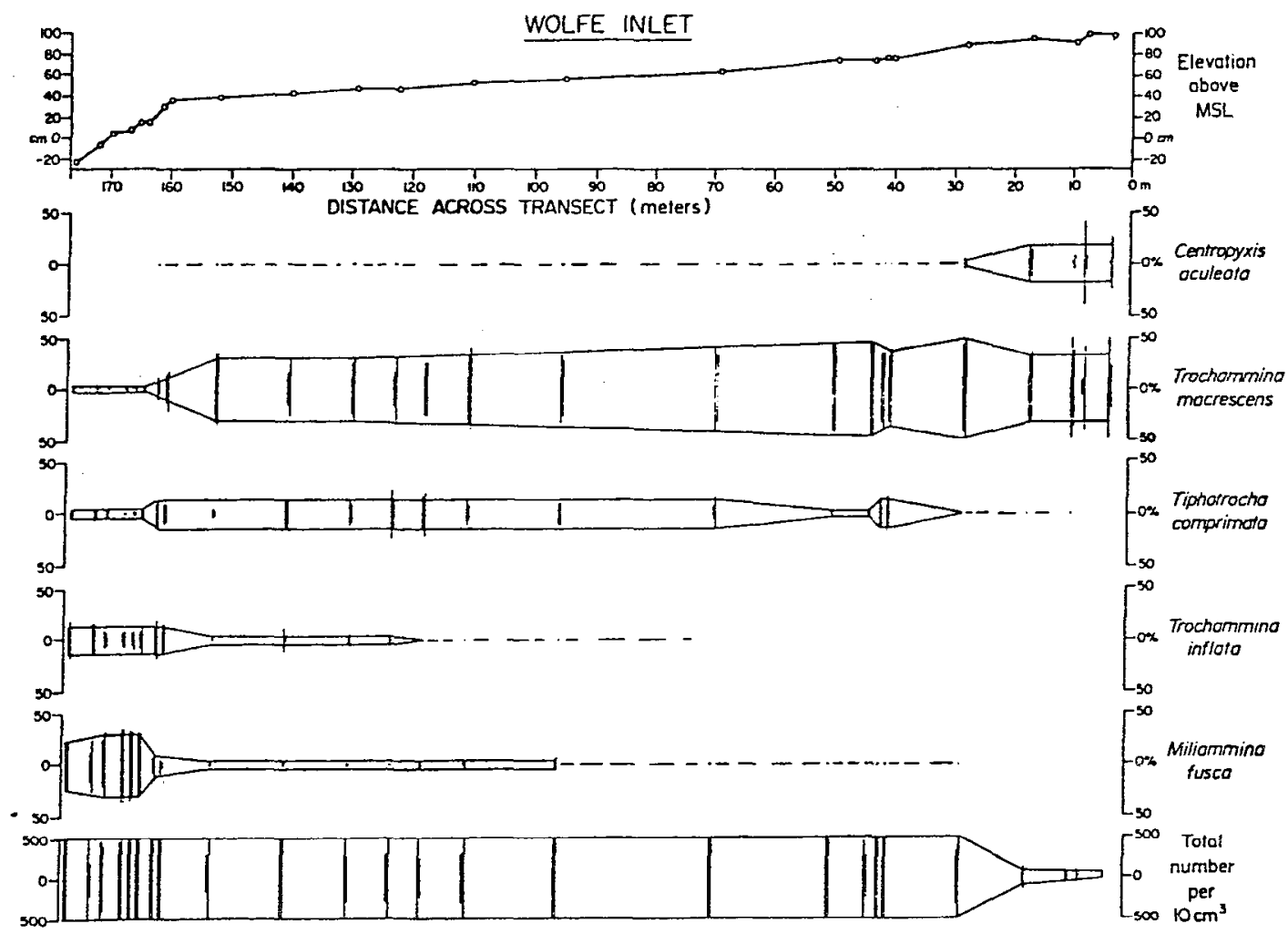

Fig. 8 - Foraminiferal distributions along Wolfe Inlet transect: Open circles are sampling localities; MSL - Benchmark MSL; double vertical bars represent the replicate samples at each locality; horizontal lines are subjective averaging (hence the vertical bars do not always fit perfectly); and, total numbers are only shown up to $1000 / 10 \mathrm{~cm}^{3}$ since all significant variations occur below this value.

the low marsh end), where a zone IIB would normally occur, T. macrescens and $T$. comprimata (but notably not $P$. (imnetis) again become dominant. Iiving populations in this area (stations 23-28, Fig. 14, Table 7.) become lower as percentages of $T$. 'macrescens increase. The slope of the channel and its sinuous nature (Figs. 5, 10) suggest that low marsh sediments are probably composed of a mixture of sediments from higher elevations, transported to lower elevations by bank undercutting and reworking.

Percival River transects: because this locality was isolated, a detailed transect with elevations was not possible. However, surface samples were collected in four semi-quantitative (without transects (Fig. 6) both to reveal differences between this area and the nearest detailed transect (Wolfe Inlet) and to detect possible spatial changes between transects (Tables 8,9 ).

As in other locations the foraminiferal distributions divide into two faunal zones with attendant subzones. The low numbers of individuals recorded at stations $1 \mathrm{~A}, \mathrm{~B} ; 2 \mathrm{~A}, \mathrm{~B} ; 3 \mathrm{~A}$ and $4 \mathrm{~A}$ indicate supra-tidal conditions. There were no thecamoebians in these samples in contrast with similar areas of the Wolfe Inlet and Mt. Stewart transects. Just below these sites zone IA is rep- 


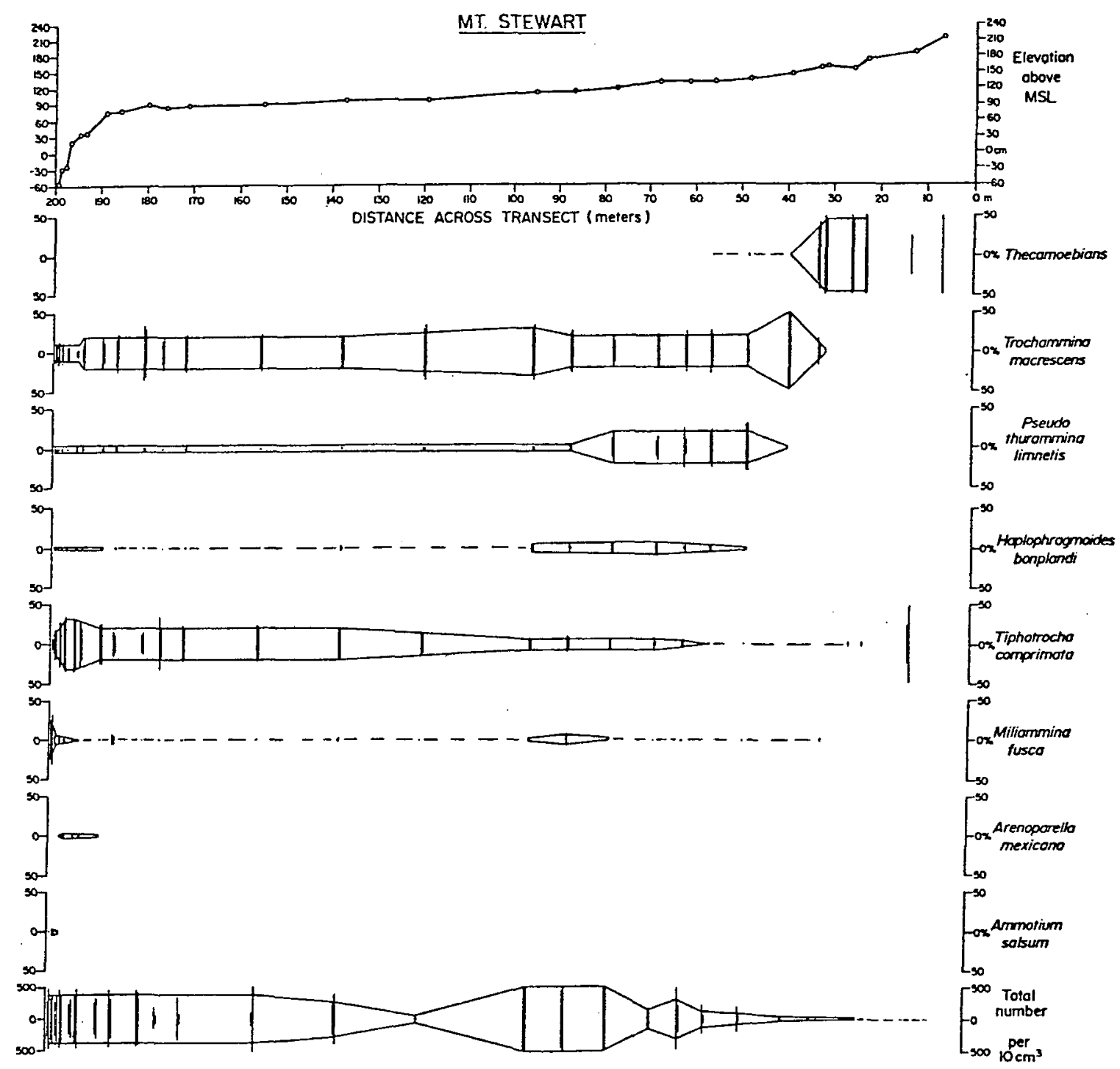

Fig. 9 - Foraminiferal distributions along Mt. Stewart transect; format same as Fig. 8.

resented at stations $1 C, D ; 2 C, D ;$ $3 \mathrm{~B}, \mathrm{C}$; and $4 \mathrm{~B}, \mathrm{C}$. Increasing percentages of Tiphotrocha comprimata and Trochammina inflata mark the occurrence of zone IB. Zone IIA occurs in samples at lower elevations which are characterized by increased $T$. inflata and Miliammina fusca. Zone IIB occurs only in the lowest samples of transects 1-3 (none in transect 4), marked by increased $M$. fusca.

These faunas are virtually identical to those in Wolfe Inlet, particularly with regard to the reduced occurrences of Pseudothuram- mina limnetis. Occurrences of $M$. fusca vary spatially, decreasing significantly at transect 4. This is consistent with changing floral composition and lower salinites of the transects going from 1 to 4 (Table 1).

Transect results from the three main areas are summarized in Figure 11 .

Foraminiferal Results in Drizl Holes and Sea Level Results

Percival River: Two drill holes were located here (Fig. 6) and 


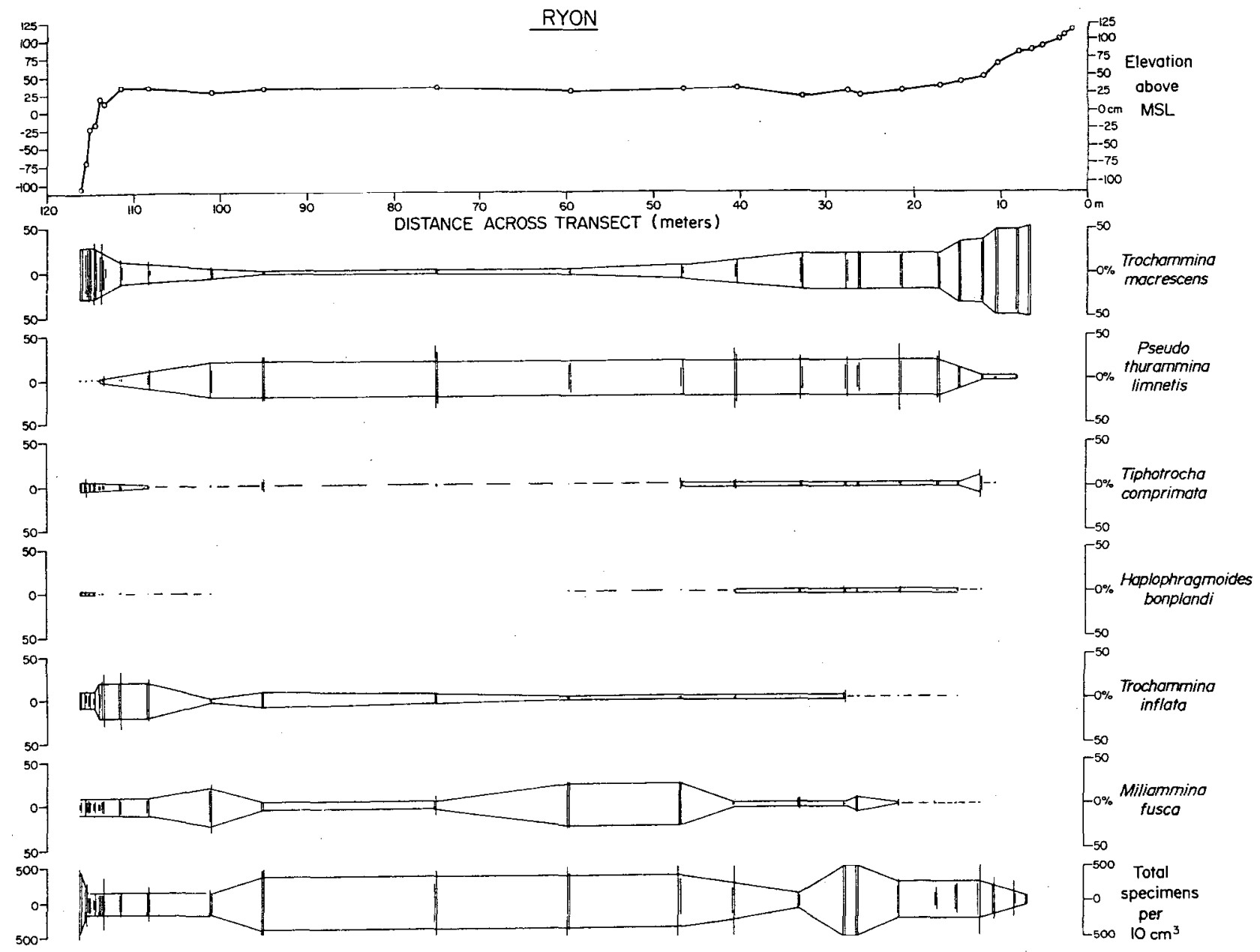

Fig. 10 - Foraminiferal distributions along Tryon transect; format same as Fig. 8.

exhibited sequences of continual marsh deposition (Fig. 12, Table 10). The faunal succession in both bore holes was similar but compressed in the shallower D.H. II. Trochammina macrescens has peak abundances near the base of both drill holes, indicating the elevation range of Zone 1A. The abundance of $T$. macrescens decreases towards the surface, accompanied by increase of Tiphotrocha comprimata and Trochammina inflata. An increase in T. inflata together with decreasing $T$. macrescens indicates the surface of the drill holes within Zone IIA.

Tryon: This wás one of the thickest peat sequences observed on P.E.I. and four drill holes were located to cover adequately the entire range of sea level rise (Fig. 5). As in the surface transect, foraminiferal numbers were lower in these drill holes than might be expected. As a result foraminiferal distributions revealed the marsh zones less clearly (Fig. 13, Table 11). Towards the base of most boreholes, lower total numbers, together with dominance of Trochammina macrescens indicate a zone I fauna. The presence of Tiphotrocha comprimata here probably places these basal samples within the upper part of Zone IB. The absence of Pseudothurammina limnetis in subsurface samples greatly limits interpretation here. 

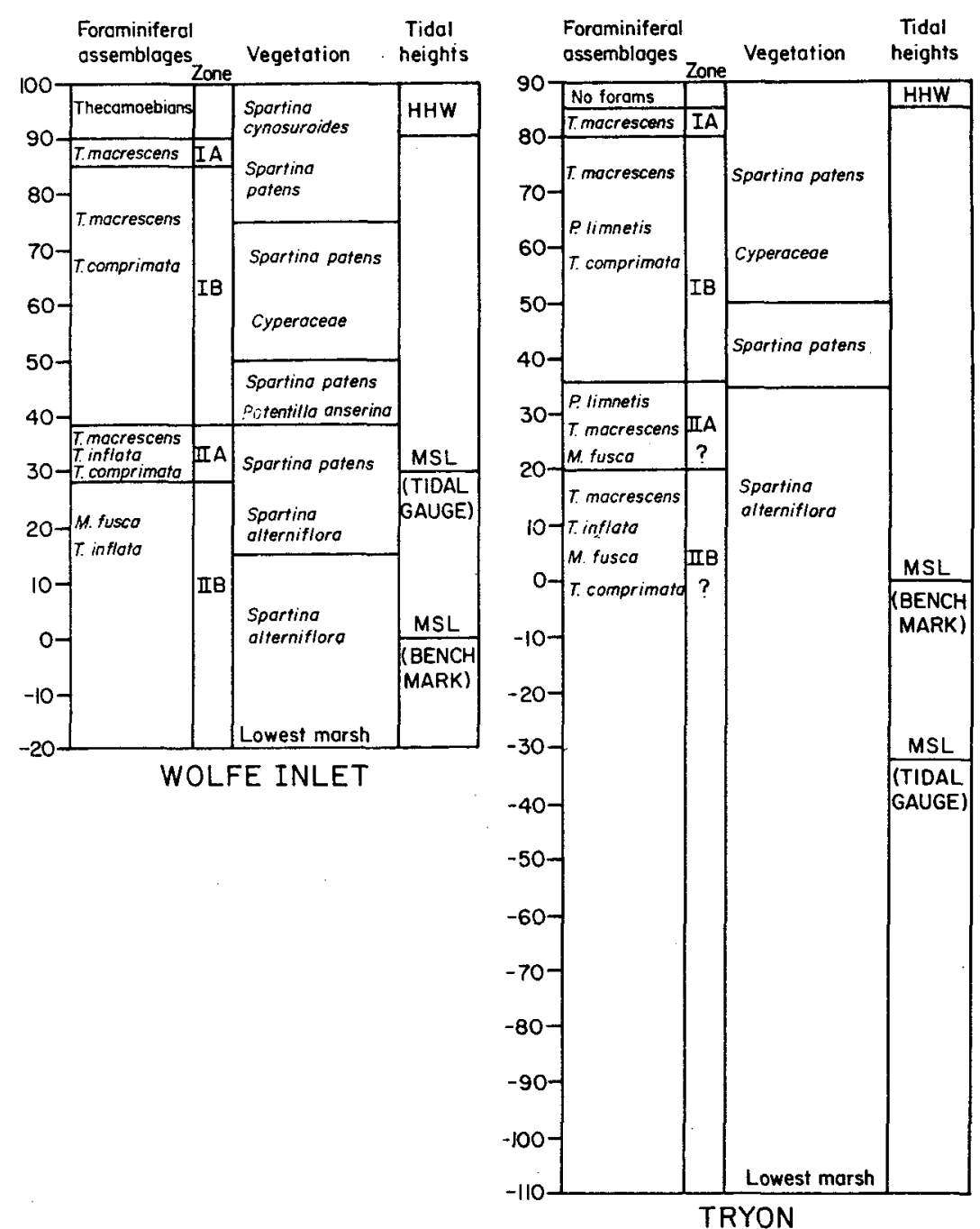

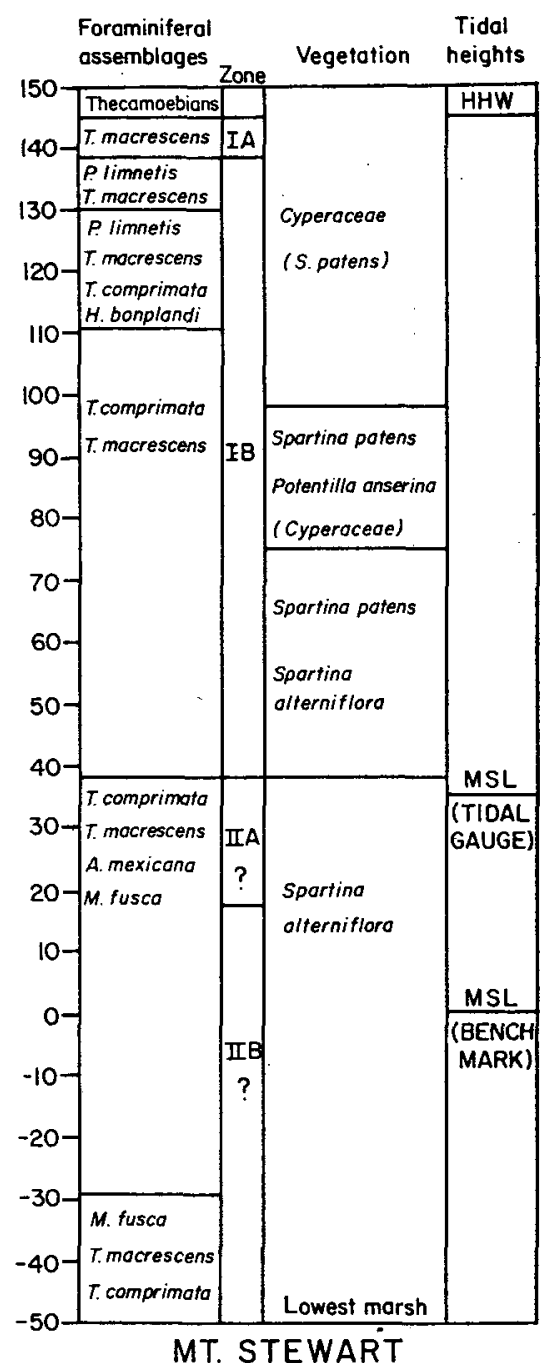

Fig. 11 - Summary diagram of the transect data. Note position of tidal MSL vs Benchmark MSL. Also note that most complicated patterns occur in the two transects located near causeways.

Pisquid: Three drill holes were located at Pisquid to cover just over $3 \mathrm{~m}$ of peat thickness (Figs. 4, 14). Foraminiferal distributions suggest a complete marsh sequence (Fig. 14, Table 12). Foraminiferal zone IA occurs near the base of the three drill holes (monospecific in Trochammina macrescens). Abundances of T. macrescens decrease tcrards the surface together with-increases in Tiphotrocha comprimata and indicate Zone IB faunas. Again Pseudothurammina limnetis is absent in subsurface sediments.

Orwell: Three drill holes were located here (Fig. 7) to encompass sea level changes recorded in just over $3 \mathrm{~m}$ of peat. Here again foraminiferal distributions revealed uninterrupted marsh sequences (Fig. 15, Table 13). In all three boreholes Trochammina macrescens dominates at the base but the presence of $T i$ photrocha comprimata suggests these deposits formed near the top of Zone IB. Towards the surface $T$. comprimata increases with a corresponding decrease in T. macrescens. Just below the surface, Miliammina fusca and Trochammina inflata show peaks and may indicate zone IIA. However, 

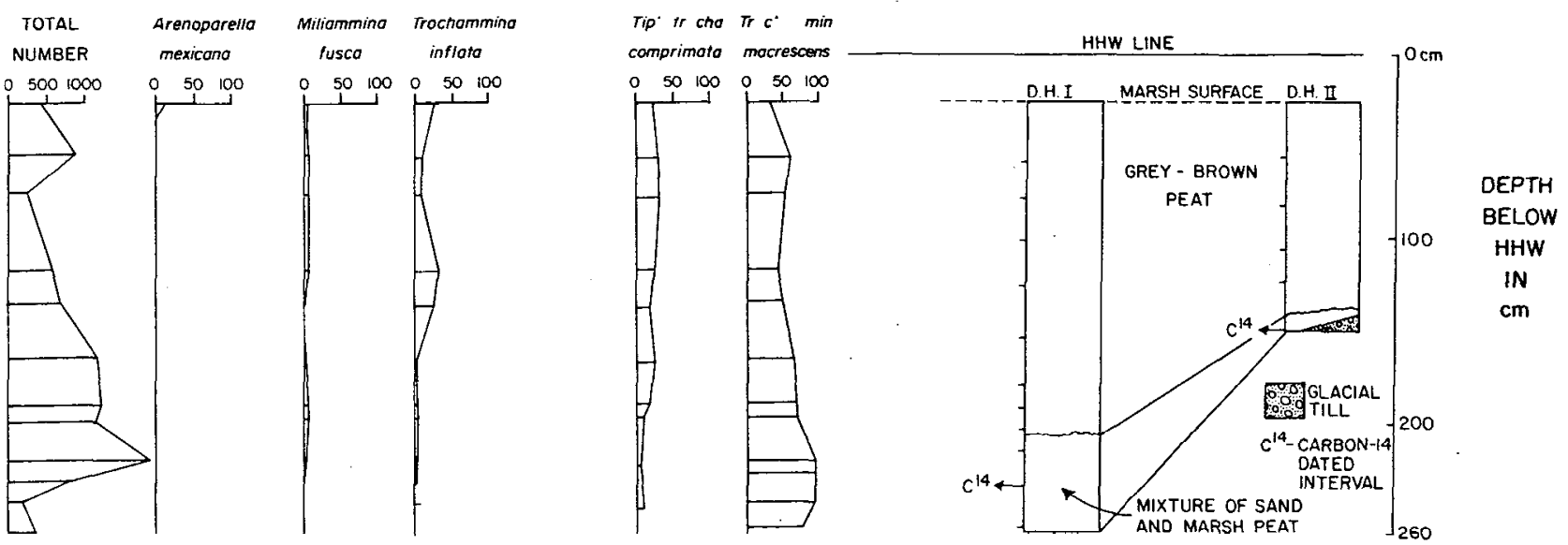

Fig. 12 - Litho- and biostratigraphy of Percival River drill holes. Only the deepest drill hole biostratigraphy is illustrated here and in subsequent figures.

high percentage occurrences of Pseudothurammina 2 imnetis indicate Zone IB again at the surface.

As indicated earlier tidal ranges at the different study sites are not the same and this affects the position of HHW but not mean sea level (MSL). If tidal range did change through time, HHW could conceivably move more or less (depending on how tidal range changed) than MSL, and not truly represent sea level change. Since we are using HHW indicators, this is an important factor. However, tidal range deviations usually require substantial changes in basin configurations which could not be generated by the relatively modest changes in relative sea level recorded here $(3-4 \mathrm{~m})$. Hence, we make the assumption that tidal ranges have remained constant throughout the last 3000 years at our study sites, and that the movement of HHW truly represents relative sea level change over this period.

Relative sea level curves: Carbon-14 dates were obtained from indicated core depths (Figs. 1215). At some sites, because of foraminiferal contents, dated levels were not located at the non-compactible substrate but were always within $20 \mathrm{~cm}$ of the base. Also, at some indicated levels, no Carbon-14 date was obtained because the sample submitted contained too little organic carbon to yield a reliable date.

These data indicate an increasing rate of relative sea level rise from west to east (Fig. 16). The Percival River data show an average of $8 \mathrm{~cm} /$ century relative sea level rise over the last 3000 years. Tryon (disregarding the dates from D.H.I. and II) indicates an average rate of $9 \mathrm{~cm} /$ century over the last 3300 years. In drill holes I and II at Tryon, dates appear anomalously young; this is probably the result of reworked materials at the base of drill holes I and II, much like the seaward end of the Tryon transect. In this case, the younger material could have come from higher elevations and been deposited in the channel bottom, giving the illusion of younger material below older material. Foraminiferal information from these drill holes also indicate this could be the problem. At 
TRYON DRILL HOLES
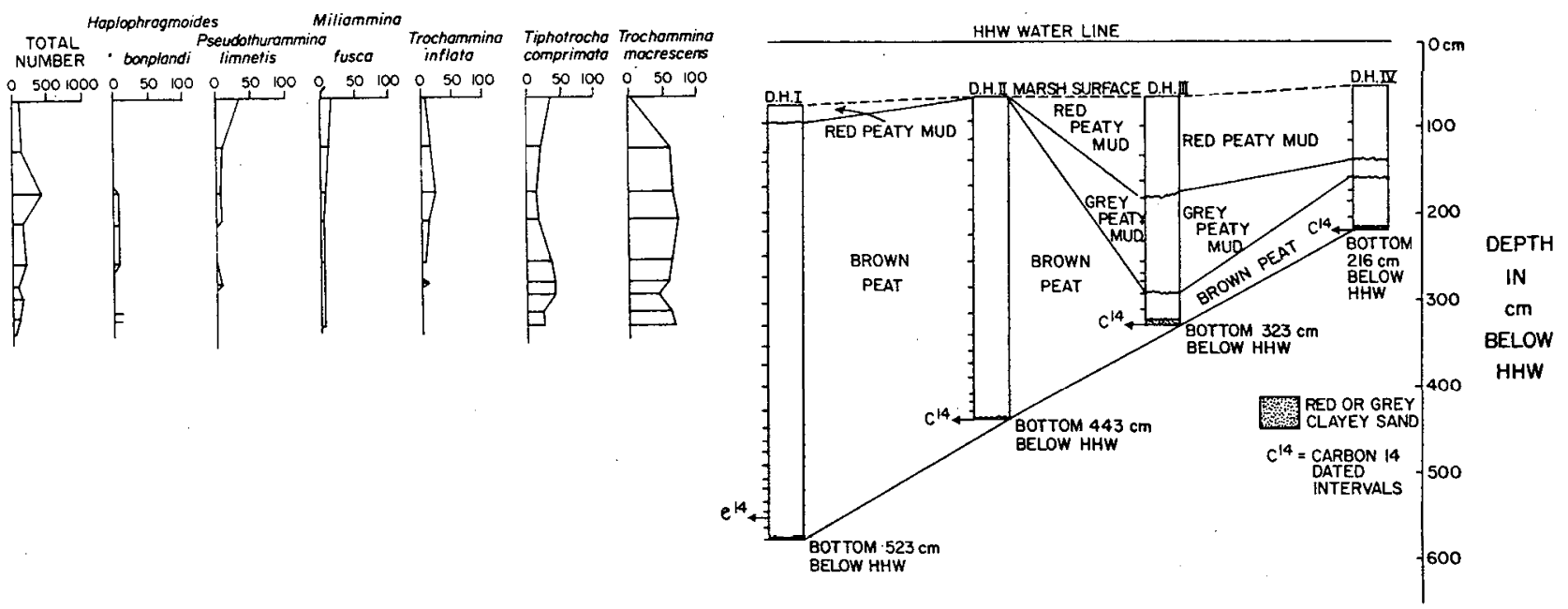

Fig. 13 - Litho- and biostratigraphy of Tryon drill holes (shallower drill hole stratigraphy illustrated since only shallow hole $\mathrm{C}^{14}$ dates were used to construct sea leve1 curve).

PISQUID DRILL HOLES
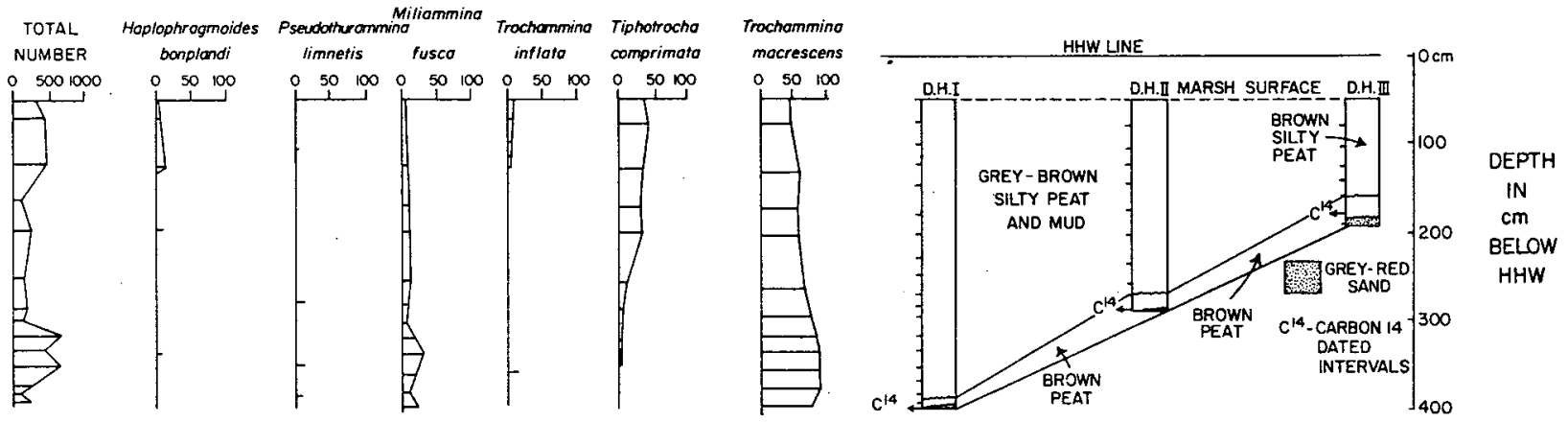

Fig. 14 - Litho- and biostratigraphy of Pisquid drill holes.

ORWELL DRILL HOLES
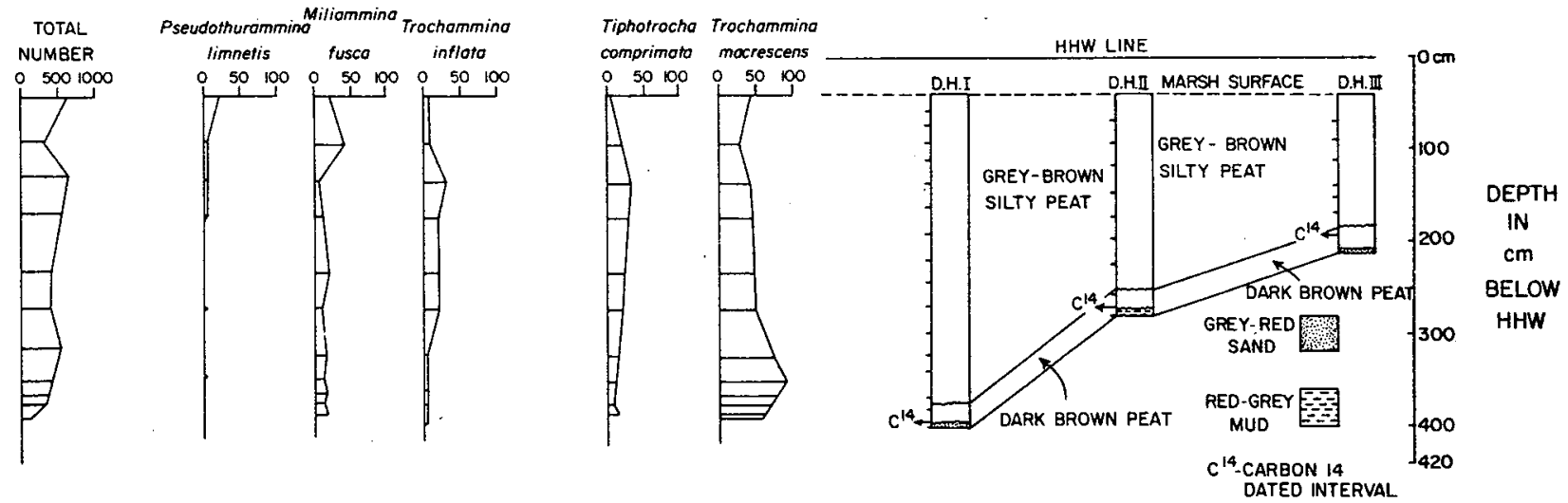

Fig. 15 - Litho- and biostratigraphy of Orwell drill holes. 
YEARS BEFORE PRESENT

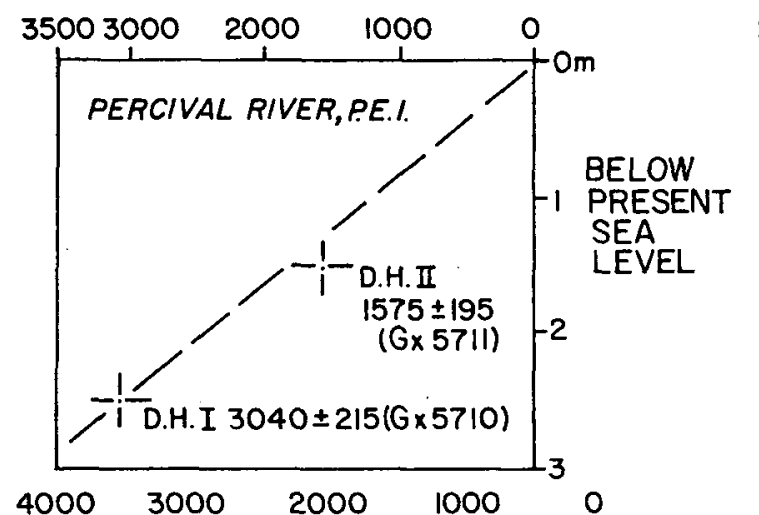

YEARS BEFORE PRESENT

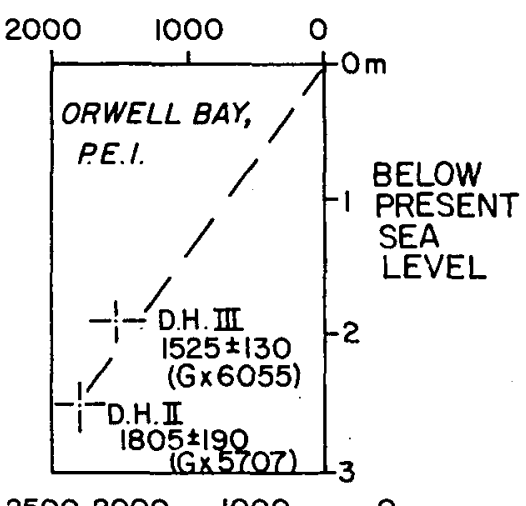

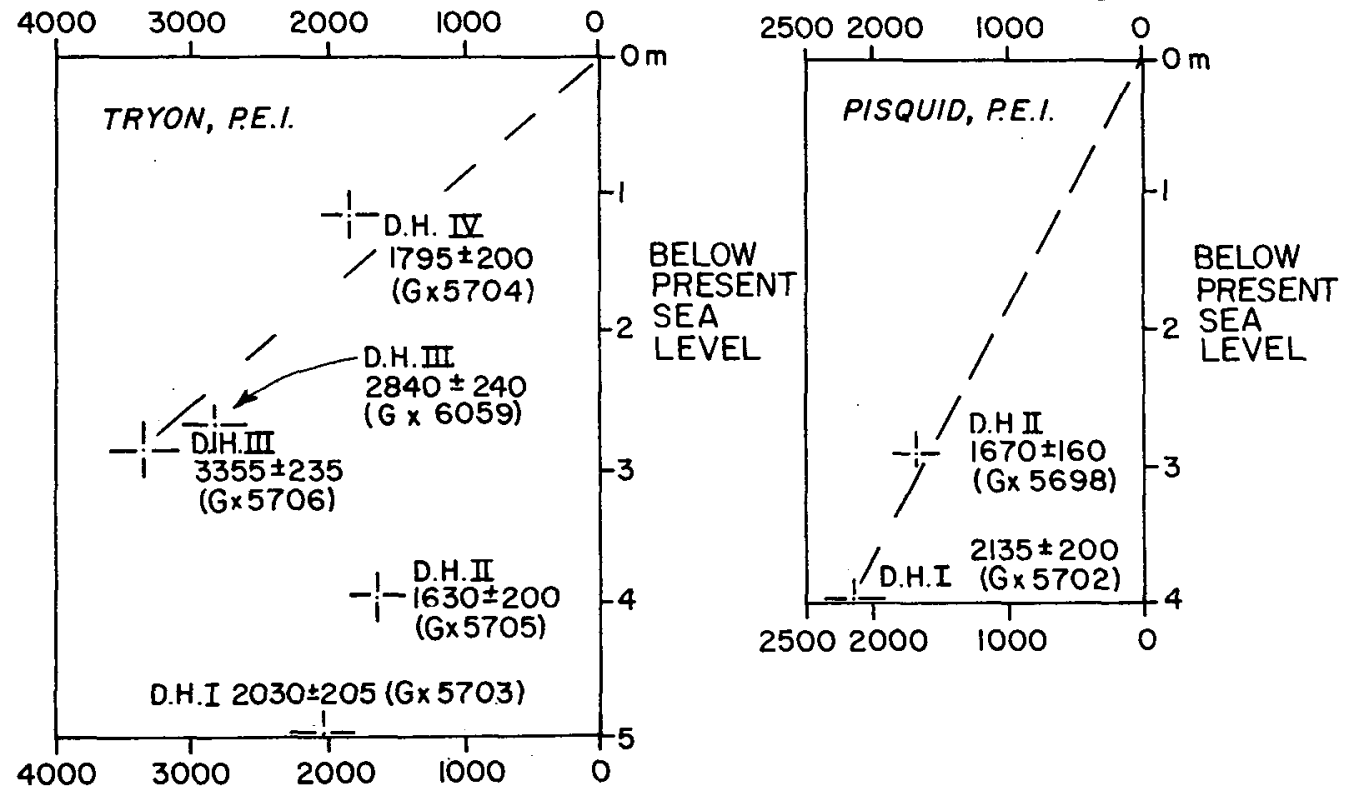

Fig. 16 - Sea level curves derived from drill holes in each of the four areas. $\mathrm{C}^{14}$ dates and lab numbers are included on the diagram. Vertical and horizontal bars indicate error limits of time and elevation.

Orwell, an average relative sea level rise of $14 \mathrm{~cm} /$ century is observed for the last 1800 years and at Pisquid, an average of 19 $\mathrm{cm} /$ century is recorded for the last 2100 years.

\section{DISCUSSION}

Surface plant relationships: Although plant distribution appears to be controlled in part by elevation above MSL, this distribution is inconsistent between areas in the same region. Several plant species have varying distributions depending, apparently, on several factors, only one of which is elevation above mean sea level. For example, Spartina patens is restricted to a narrow elevation range in Atlantic Coast marshes but dominates at all but the lowest levels (including some supra-tidal areas) in P.E.I. marshes. Oddly, in Wallace Basin, Nova Scotia (just across the strait from P.E.I.)S. patens again shows a restricted distribution (Scott and Medioli, 1980a). Differing elevational ranges for this species and others in adjacent areas significantly reduce vertical resolution of deposits using only plant remains. Hence, even if plant remains were easily recognizable in ancient marsh deposits, 
their reliability as sea level indicators would be low relative to those of foraminifera.

Surface foraminiferal distributions: There are some individual characteristics of the P.E.I. foraminiferal faunas that warrant special discussion. Pseudothurammina limnetis, a form described from Nova Scotia but never a dominant species there, appears to replace Tiphotrocha comprimata in Zone IB faunas of P.E.I. marshes where tidal range exceeds $2 \mathrm{~m}$. However, $P$. limnetis does not appear in subsurface sediments or reworked sediments (low marsh area of Tryon); this suggests that tests of this species do not preserve once the organism dies. As noted in the type description of this species (Scott and Medioli 1980 a) the test is flexible and derives its strength from an organic inner lining rather than the cement of the agglutinated material covering the lining. Apparently the inner lining of this species, unlike inner pseudochitinous linings of other marsh species, is not resistant to decay and destruction in the highly bacteriologically active marsh sediments. Hence, although this species is common in some marshes, it is less useful than other species for paleoecological and sea level studies.

Two of the transect localities (Tryon, Mt. Stewart) were located near causeways. In Tryon the transect was just landward of the causeway (Fig. 5) and at Mt. Stewart the transect was just seaward (Fig. 4). It is difficult to assess the impact of the causeways on tidal ranges, circulation patterns, and the living marsh assemblages without having precise measurements of those elements, both before and after the causeways were constructed. However, comparing the Tryon and Mt. stewart data with other marsh areas, most marked differences occur in the low marsh assemblages (i.e. between mid-tide and $3 / 4$ tide). Additionally, distribution patterns observed at these two transects are among the most complex recorded from any marsh. Hence, we must conclude that those areas are affected in a complex manner and must be considered as abnormal systems, not comparable with unrestricted marsh systems, such as those studied in Nova Scotia or Wolfe Inlet and Percival River in this report. It is not the intent of this paper to discuss in detail what changes may have occurred as a result of causeway placements; however, it appears that a study of this kind could be initiated using as a starting point, data presented here and working back in time by means of drill holes or cores.

Characteristic of marshes examined in P.E.I. is the complete absence of calcareous species. This is unexpected since the shallow estuarine environments studied by Bartlett and associates are dominated by calcareous species. One calcareous species was recorded (mostly living specimens) but specimens lacked a carbonate test; only the organic inner lining was observed (Ammonia beccarii). Although salinites and temperatures in both Nova Scotia and P.E.I. marshes are similar, the areas physiographically are noticeably different. Areas investigated in Nova Scotia were large, open areas with high tidal turbulence while marshes examined in P.E.I. were in areas where channels were relatively small and turbulence reduced. The reduction of turbulence probably decreases the amount of dissolved oxygen in tidal waters. This in turn would depress $\mathrm{pH}$ levels at high tide when they are normally raised by high dissolved $\mathrm{O}_{2}$ in flood tides (Phleger and Bradshaw 1966). Hence, 
TABLE 10

FORAMINIFERAL PERCENTAGE OCCURRENCES IN PERCIVAL RIVER DRILL HOLES

$$
\text { D.H.I. } 0-230
$$

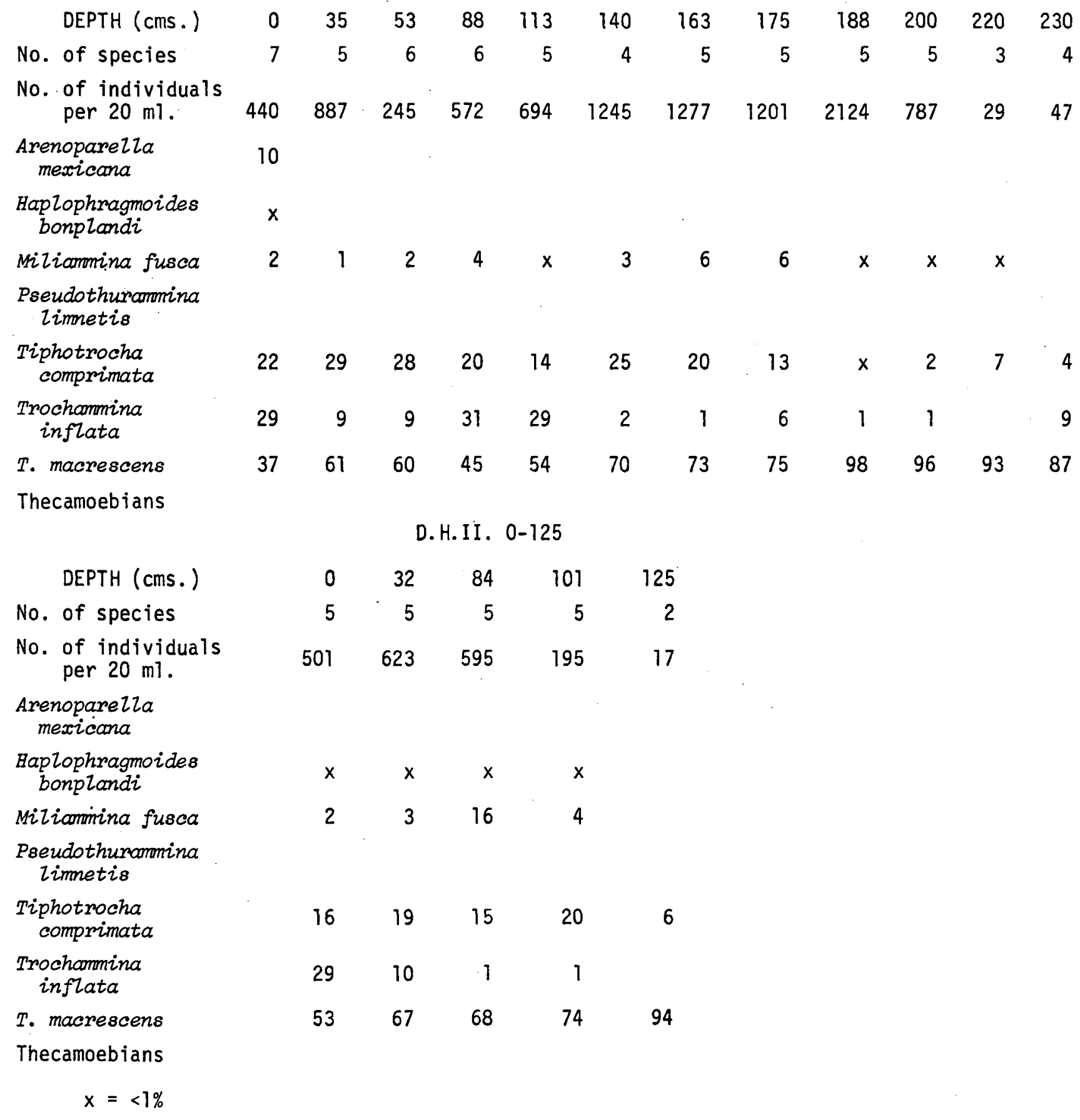

although salinities may be sufficiently high, $\mathrm{pH}$ may be the limiting factor for the calcareous species. An exact parallel was observed in California where Mission Bay marshes, flooded by tur- bulent waters of an open bay, were dominated by calcareous species in low marsh areas; only $30 \mathrm{~km}$ south (Tiajuana slough) marshes with similar salinities but restricted flow, were dominated by 
TABLE 11

FORAMINIFERAL PERCENTAGE OCCURRENCES IN TRYON DRILL HOLES

D.H.I.

\begin{tabular}{|c|c|c|c|c|c|c|c|c|c|c|c|c|c|c|c|}
\hline DEPTH (cms.) & 0 & 20 & 65 & 123 & 173 & 228 & 278 & 323 & 373 & 400 & 432 & 459 & 476 & 487 & 503 \\
\hline No. of species & 6 & 5 & 4 & 4 & 5 & 5 & 6 & 6 & 5 & 5 & 5 & 5 & 5 & 4 & 3 \\
\hline $\begin{array}{l}\text { No. of individuais } \\
\text { per } 20 \mathrm{ml} \text {. }\end{array}$ & 274 & 460 & 170 & 154 & 372 & 177 & 572 & 700 & 612 & 664 & 396 & 284 & 222 & 156 & 178 \\
\hline \multicolumn{16}{|l|}{ Arenoparella mexiccana } \\
\hline Haplophragmoides bonplandi & 2 & & & 4 & $x$ & 0 & 2 & 1 & $x$ & 1 & 1 & $x$ & & & \\
\hline Miliammina fusca & 15 & 20 & 7 & 3 & 9 & 7 & 8 & 6 & 7 & 5 & 7 & 5 & 23 & 13 & 4 \\
\hline Pseudothuranmina limnetis & 28 & 20 & & & & 1 & 2 & 2 & $x$ & 2 & 3 & 4 & 3 & $x$ & \\
\hline Tiphotrocha comprimata & 5 & 10 & 14 & 6 & 4 & 7 & 8 & 6 & 5 & 20 & 7 & 14 & 6 & 12 & 5 \\
\hline Trochammina inflata & 7 & 39 & 1 & & $x$ & 1 & 7 & 1 & & & & & & & \\
\hline T. macrescens & 43 & 11 & 78 & 87 & 86 & 84 & 79 & 84 & 87 & 72 & 82 & 76 & 66 & 74 & 91 \\
\hline
\end{tabular}

Thecamoebians

D.H.II.

DEPTH (cms.)

No. of species

No. of individuals per $20 \mathrm{ml}$.

Arenoparella mexicana Haplophragmoides bonplandi Miliamina fusca Pseudothuramina limnetis Tiphotrocha comprimata Trochanmina inflata T. macrescens $\begin{array}{lllllllll}95 & 223 & 253 & 296 & 307 & 323 & 353 & 363 & 375\end{array}$ Thecamoebians

$\begin{array}{rrrrrrrrrrrr}0 & 76 & 133 & 195 & 223 & 253 & 296 & 307 & 323 & 353 & 363 & 375 \\ 5 & 6 & 4 & 6 & 5 & 5 & 5 & 4 & 6 & 5 & 7 & 2 \\ 62 & 127 & 82 & 141 & 122 & 146 & 121 & 139 & 168 & 166 & 195 & 44\end{array}$

$\begin{array}{rrrrrrrrrrrrr}19 & 5 & 23 & 1 & 2 & 2 & 1 & 1 & x & 1 & 2 & 5 \\ & 9 & & 8 & 2 & 12 & 8 & 3 & 6 & 12 & 6 & \\ 4 & 4 & & 3 & & 2 & & & 4 & 1 & 3 & \\ 10 & 8 & 13 & 25 & 17 & 12 & 11 & 4 & 8 & 17 & 23 & \\ 37 & 8 & 34 & 7 & 13 & & 1 & & 3 & & \times & \\ 30 & 66 & 30 & 56 & 66 & 72 & 79 & 92 & 79 & 69 & 65 & 95 \\ & & & & & & & & & & \times & \end{array}$

\section{D.H.III.}

DEPTH (cms.)

$\begin{array}{lllllllll}0 & 53 & 103 & 132 & 186 & 210 & 223 & 242 & 263\end{array}$

No. of species

No. of individual per $20 \mathrm{ml}$.

Arenoparella mexicana Haplophragmoides bonplandi Miliamina fusca Pseudothuramina limmetis Tiphotrocha comprimata Trochammina inflata

T. macrescens

Thecamoebians

$\begin{array}{rrrrrrrrr} & x & & & & & 1 & 7 & \\ 15 & 10 & -1 & 2 & 3 & 7 & 5 & 5 & 2 \\ 34 & 1 & x & 3 & & 1 & 4 & & \\ 39 & 23 & 13 & 17 & 33 & 30 & 40 & 23 & 25 \\ 2 & 5 & 16 & 2 & x & & 1 & & \\ 10 & 61 & 68 & 74 & 63 & 62 & 49 & 65 & 73 \\ & & & x & & & & & \end{array}$

D.H.IV.

DEPTH (cms.) $\begin{array}{llllllll}0 & 53 & 88 & 105 & 120 & 134 & 153 & 168\end{array}$

No. of species

No. of individual

per $20 \mathrm{ml}$.

Arenoparella mexicana Haplophragmoides bonplandi Milianmina fusca Pseudothuromina limetis Tiphotrocha comprimata Trochamina inflata

T. macrescens

Thecamoebians $x=<1 \%$ 
TABLE 12

FORAMINIFERAL PERCENTAGE OCCURRENCES IN PISQUID DRILL HOLES

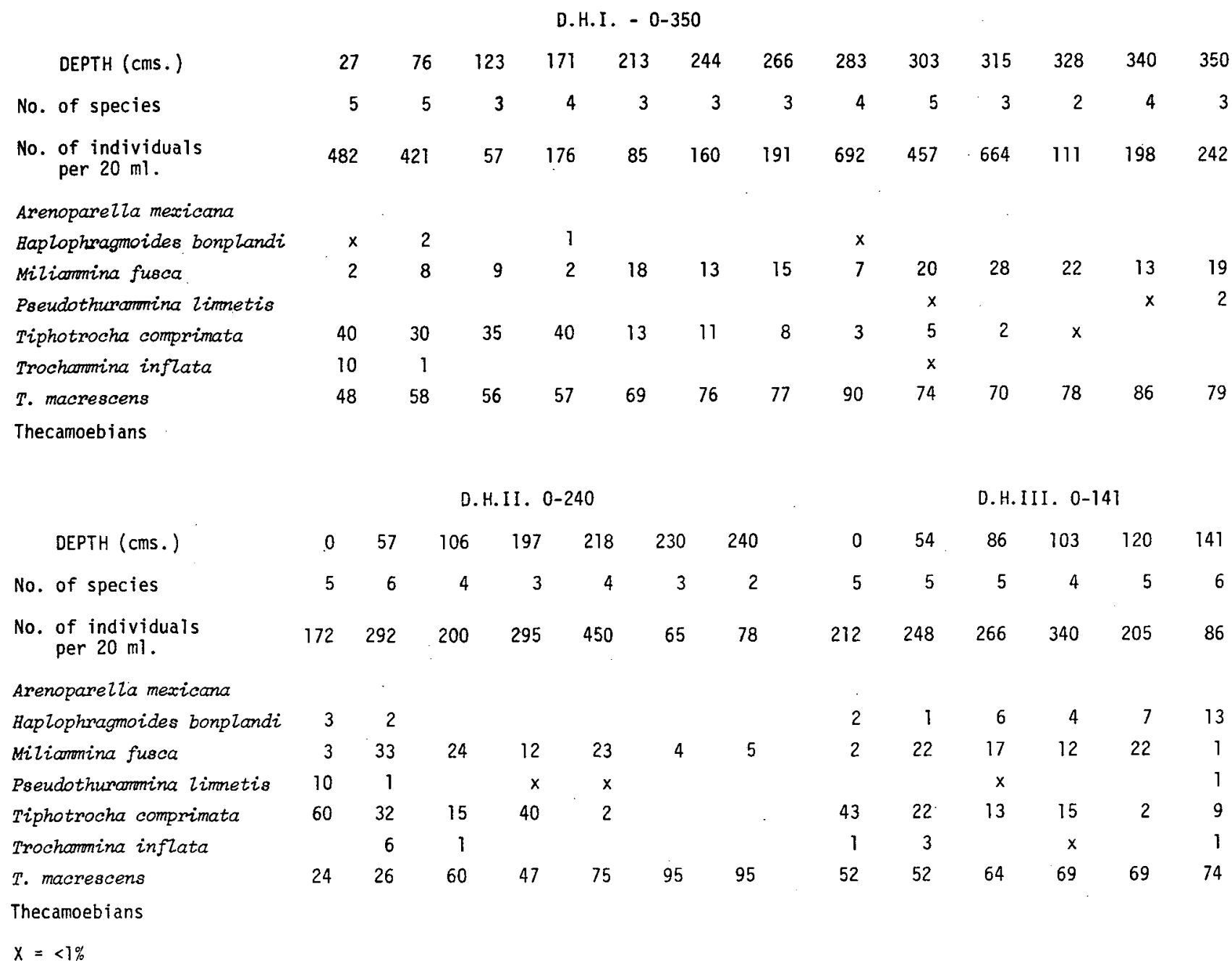

arenaceous species (Scott 1976 b).

Vertical tidal relationships: There appears to be a discrepancy between tidal datum (MSI or $\mathrm{Z}_{0}$ ) and the datum (given as MSL) used for benchmarks. Tidal MSL occurs consistently above the mid-tide level while it appears that midtide was used as MSL for the benchmarks. It is noteworthy, however, that marsh distributions, both vegetation and foraminifera, align as if MSL was at the midtide level. However, the middle and high plant zones and corresponding foraminiferal zones encompass a slightly broader ele- vational range than parallel zones in a non-mixed tidal system. Scott and Medioli (1980 a) suggested that these two zones are usually confined to the upper $30-40 \mathrm{~cm}$ of tidal range (sea level accuracy of $\pm 15-20 \mathrm{~cm}$ ), regardless of tidal amplitude. These same zones in P.E.I. marshes occupy up to $75 \mathrm{~cm}$ total range (Mt. Stewart) or the upper $\frac{1}{4}$ of the tidal range which gives them an accuracy of $\pm 30-40$ $\mathrm{cm}$. This is also true in Wallace Basin marsh, Nova Scotia (a point overlooked by scott and Medioli 1980 a). Hence, although zone I species are still restricted to the upper $\frac{3}{4}$ of tidal range (Scott 
TABLE 13

FORAMINIFERAL PERCENTAGE OCCURRENCES IN ORWELL DRILL HOLES

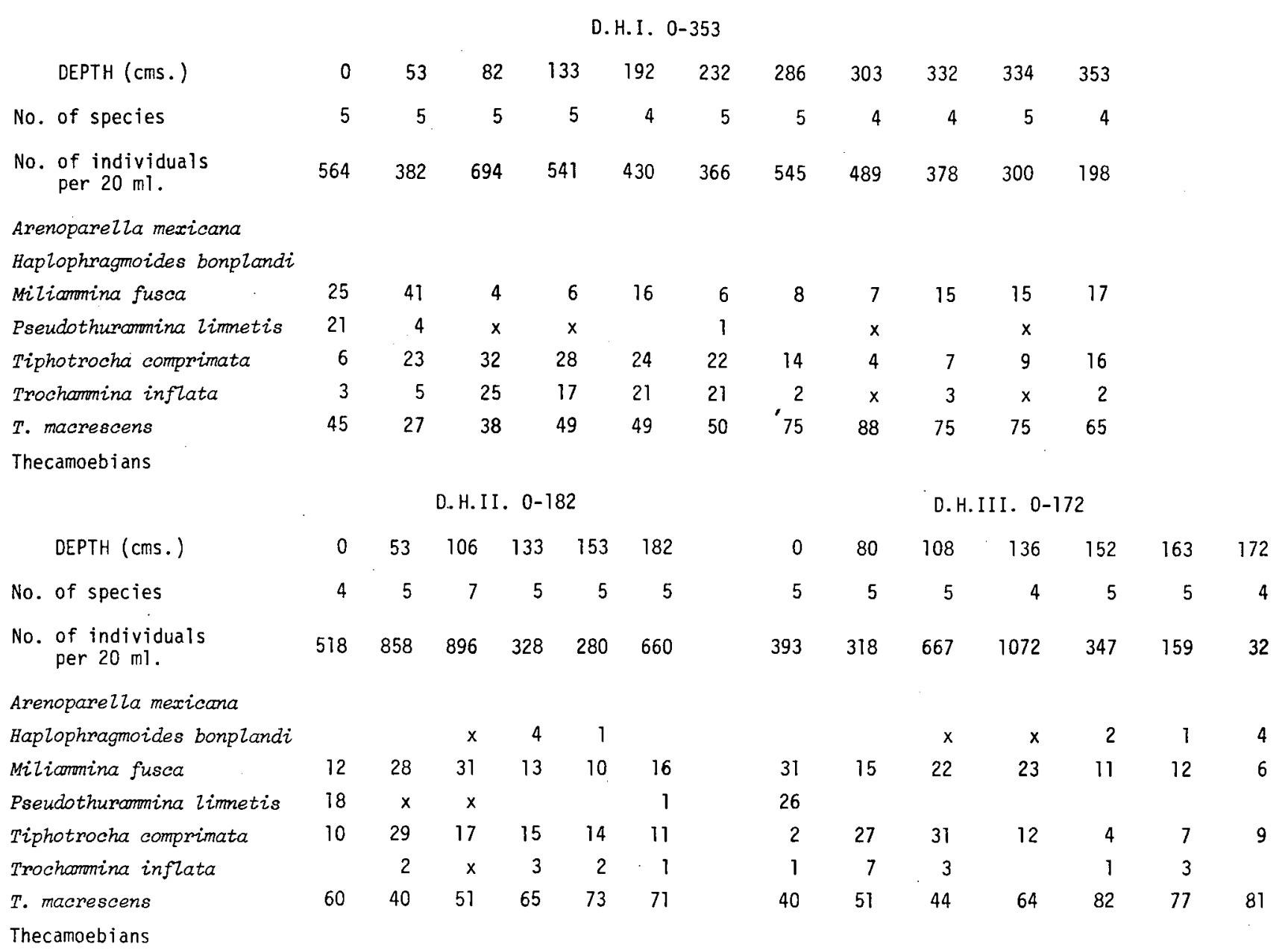

$X=<\uparrow \%$

and Medioli $1978 \mathrm{~b}$ ), their absolute vertical range apparently increases in response to the mixed tidal system. This however, does not appear to affect the absolute range of zone IA (i.e. the $T$. macrescens zone), which even in P.E.I. still retains its absolute accuracy of $\pm 5 \mathrm{~cm}$.

Sea level changes: Orwell is the only area in this study close to sites where previous onshore sea level data are available. Frankel and Crowl (1961) report a range of 1.5 to $2.4 \mathrm{~m}$ of relative sea level rise in the last 900 years at Nicholas Point, about $20 \mathrm{~km} \mathrm{SE}$ of orwell; this is in contrast to
$1.25 \mathrm{~m}$ over the same period recorded at Orwell (Fig. 16). The range of values inaicated by Frankel and Crowl (1961) is probably the result of their use of less precise indicators of former sea level (tree stumps, undifferentiated peat), with their lower value ( $1.5 \mathrm{~m} / 900$ yrs) being closest to our relative sea level change.

The general trend of decreasing sea level rise westward was reported by Kranck (1972) from offshore studies but she suggested that, for the last 3000 years, all of P.E.I. has experienced uniform relative sea level rise. Our 
data suggest that the rate of rise has been almost twice as fast in the east than at the western end of P.E.I. Kranck (1972) acknowledged that her data were too limited to determine when differential movement terminated. Our data also indicate that magnitudes of sea level rise, particularly in the west, estimated by Kranck (1972) were excessively high. As with Frankel and Crowl (1961) the use of less precise indicators (in this case miscellaneous shells) probably caused the discrepancy in Kranck's (1972) sea-level figures.

Unfortunately, the lack of thicker marsh deposits limits the sea level record to only the last 3000 years. However, these data were still useful in calibrating the geophysical models of relative sea level movement presented by Quinlan and Beaumont (1981). We have also demonstrated that marsh foraminiferal zonations can be used to detect small scale differences of sea level change, which was not previously possible.

\section{ACKNOWLEDGEMENTS}

We are indebted to many people and several agencies for making this work possible. P.J. Mudie and G. Quinlan (Dalhousie University) critically read the manuscript. I. McQuarrie (U.P.E.I.) advised us on marsh locations and supplied us with lab space at U.P.E.I. C. Murphy (Department of Environment, P.E.I.) provided information on tidal range in Hillsborough River; R. Duggan (Environment Canada) provided accommodation and lab space for work in Western P.E.I. C. Garrett (Dalhousie University) supplied us with tidal gauge data. The final manuscript was critically read by J.P. Guilbault and G.B. Fader of the Atlantic Geoscience Centre.
Energy, Mines, Resources, Canada and the Atlantic Geoscience Centre (D. Euckley and M. Gorveat in particular) provided logistical support in the form of a Honda ATC and trailer for transporting equipment to remote locations. Funding for the work was provided through EMR research agreement \# 2239-4-31/78 and NSERC Operating and Strategic Grants to F. S. Medioli and C. Beaumont (Da1housie).

\section{SYSTEMATIC TAXONOMY}

No synonymies or plates are included in this paper (except for the new genus). The reader is referred to Scott and Medioli $(1980$ a) for descriptions and plates of all foraminiferal species. Thecamoebian species are illustrated in scott and others (1980) with the following name aifferences - Difflugia obionga (here) $=D$. capreolata (Scott and others, 1980); Centropyxis aculeata (here) $=C$. excentricus (scott and others, 1980); Centropyxis constricta (here) = Urnulina compressa (Scott and others, 1980).

Following is the description and systematic placing of the new genus, Pseudothurammina scott, Medioli, Williamson.

Family - Saccaminidae Brady 1884 Sub-family - Saccammininae Brady 1884

Genus Pseudothurammina $n$. gen. scott, Medioli and Williamson Genotype: Thuramina? limnetis Scott and Medioli, 1980 a p. 43, 44, pl. 1, figs. 1-3

Generic diagnosis: Test free or attached, monothalamous, subglobular; variable number $(0-5$ in specimens we have observed) of irregular mammillae occur in the outer test; apertures at apex of the mammillae. Wall flexible with relatively thin layer of mineral 
grains cemented to an organic (not pseudochitinous) inner lining. Organic lining transparent, usually visible in area of attachment where there is no agglutinated material.

Ecology and occurrence: Occurrence is basically the same as reported for the type species, $P$. limnetis (Scott and Medioli 1980a). However, since that work, Dr. D. Haman (pers. comm.) has reported finding $P$. Limnetis in levee deposits (presumably in or near a marsh) from the Northeast Pass, Mississippi Delta (lat. 29०7'59", long. $89^{\circ} 2^{\prime} 12^{\prime \prime}$ ). Water depth at time of collection was $30 \mathrm{~cm}$ and he reported finding specimens to a depth of $30 \mathrm{~cm}$ in the sediment. Salinites were low $(0-6 \%$ ) and temperatures $h_{i}$ gh $\left(21^{\circ} \mathrm{C}\right)$ at time of collection, not inconsistent with summer conditions in marshes of Maritime Canada. This report, together with probable occurrences in Brazil and Europe, lead us to believe the genus has a worldwide distribution.

Remarks: Specimens belonging to this genus have previously been placed with several genera, among them Astrammina Rhumbler, Armorella Heron-Allen and Earland and Thurammina Brady. It was suggested by Scott and Medioli (1980a) that a new genus was probably in order for the species $P$. Limnetis, at that time doubtfully placed with Thurammina. Since that time we have had the opportunity to examine specimens of Thurammina species. Although these specimens had a slightly flexible test (as in Pseudothurammina) there was no inner lining and Thurammina appear to be deep water forms, as opposed to the marsh habitat of Pseudothurammina.

It was stated in the type description of $P$. Limnetis that the organic inner lining was pseudochitinous (Scott and Medioli 1980 a). However, in addition to its transparent nature, the inner lining of Pseudothurammina does not preserve in subsurface or transported sediments, unlike the pseudochitinous linings of other marsh species. The term "pseudochitinous" is a loosely defined term, applied generally to all inner linings of foraminifera; however, here we chose to differentiate the lining in Pseudothurammina from that of other foraminiferal species because of its preservation characteristics.

Pseudothurammina was placed with the family saccamminidae based on wall structure and general test form.

Generic derivation: The name Pseudothurammina was chosen because outwardly, specimens of this genus appear similar to those belonging to the genus Thurammina.

DAWSON, J.W. and HARRINGTON, B.J. 1871. Report on the geological structure and mineral resources of Prince Edward Island: Montreal, John Lovel.

DYCK, W. and FYLES, J.C. 1963. Radiocarbon dates II. Geological Survey of Canada Paper 63-21.

carbon dates III. Geological Survey of Canada Paper 64-40:

FRANKEL, L. and CROWL, G.H. 1961. Drowned forests along the eastern coast of Prince Edward Island, Canada. Journal of Geology, 69, pp. 352-357.

GESNER, A. 1846. Report of the geological survey of Prince Edward Island. Report to H.V. Huntley, Lt. Governor.

1861. On elevations and depressions of the earth in North America. Quarterly Journal of the Geological Society, London, 17, pp. 381388 .

GRANT, D. R. 1970a. Recent coastal submergence of the Maritime provinces, 
Canada. Canadian Journal of Earth Sciences, 7, pp. 676-689.

1970b. Recent coastal submergence of the Maritime provinces, Canada. Ph.D. dissertation, Corne11 University, New York, 109p.

JOHNSON, D.W. 1913a. The shoreline of Cascumpeque Harbour, Prince Edward Island. Geographical Journal, 42, pp. 152-164.

1913b. Botanical phenomena and the problem of recent coastal subsidence. Botanical Gazette, 56, pp. 449-468.

KAYE, C. A. and BAARGHORN, E. S. 1964. Late Quaternary sea-level change and crustal rise at Boston, Massachusetts, with notes on the auto-compaction of peat. Geological Society of America, Bulletin, 75, pp. 63-80.

KRANCK, K. 1972. Geomorphological development and post-Pleistocene sea level changes, Northumberland Strait, Maritime Provinces. Canadian Journal of Earth Sciences, 9, Pp. 835-844.

MEDIOLI, F. S. and SCOTT, D. B. 1976. A portable, hand operated device for drilling in soil and salt marsh deposits. Maritime Sediments, 12, pp. $77-78$.

OWEN, E.B. 1949. Pleistocene deposits of O'Leary Map-area, Prince Edward Island. Geological Survey of Canada, Paper 49-6.

PALMER, A. J. M. 1974. Diatom stratigraphy and post-glacial history of Basin Head Harbour, Prince Edward Is1and. M.Sc. Thesis, Dalhousie University, Halifax, 143p.

PELTIER, W.R. and ANDREWS, J.T. 1976. Glacial-isostatic adjustment - I. The forward problem. Geophysical Journal of the Royal Astronomical Society,46, pp. 605-646.

PHLEGER, F.B and BRADSHAW, J.S. 1966. Sedimentary environments in a marine marsh. Science, 154, pp. 1551-1553.

PREST, V. K. 1962. Geology of Tignish map-area, Prince County, Prince Edward Island. Geological Survey of Canada, Paper 61-28.

1973. Surficial deposits of Prince Edward Island. Map 1366A, Geological Survey of Canada.

QUINLAN; G.M. and BEAUMONT, C. 1981. A comparison of observed and theoretical post-glacial relative sea level in Atlantic Canada. Canadian Journal of Earth Science, 18, pp. 1146-1163.

SCOTT, D.B. 1976a. Brackish-water foraminifera from southern California and description of Polysaccammina ipohalina n. gen., n. sp. Journal Foraminifera1 Research, 6, pp. 312-321.

1976b. Quantitative studies of marsh foraminiferal patterns in southern California and their application to Holocene stratigraphic problems. 1st International Symposium on Benthonic Foraminifera of Continental Margins. Part A. Ecology and Biology, Maritime Sediments Special Publication, No. 1, pp. 153-170.

1977. Distributions and population dynamics of marsh-estuarine foraminifera with applications to relocating Holocene sea level. Ph.D. Thesis, Dalhousie University, Halifax, 252p.

SCOTT, D.B. and MEDIOLI, F.S. 1978a. Vertical zonations of marsh foraminifera as accurate indicators of former sea levels. Nature, 272, pp. 528-531.

Studies of relative sea level changes in the Maritimes. Progress Report to Energy, Mines, Resources, Canada, Report No. EMR 2239-4-31/78, 79p.

Quantitative studies of marsh foraminiferal distributions in Nova Scotia: implications for sea level studies. Cushman Foundation for Forminifera1 Research, Special Publication No. 17, $58 \mathrm{p}$.

Living vs. total foraminiferal popu- 
lations: their relative usefulness in Paleoecology. Journal of Paleontology, 54, pp. 814-831.

SCOTT, B.D., SCHAFER, C.T. and MEDIOLI, F.S. 1980. Eastern Canadian estuarine foraminifera: a framework for comparison. Journal of Foraminiferal Research, 10, pp. 205-234.

\author{
Reviewers: G.B. Fader \\ J.P. Guilbault \\ B.R. Pelletier
}

